ARTICLE

\title{
Smad3 promotes cancer progression by inhibiting E4BP4-mediated NK cell development
}

\author{
Patrick Ming-Kuen Tang ${ }^{1,2, \star}$, Shuang Zhou ${ }^{2,3,4, \star}$, Xiao-Ming Meng ${ }^{2, \star}$, Qing-Ming Wang ${ }^{2}$, Chun-Jie Li ${ }^{2}$, \\ Guang-Yu Lian ${ }^{2}$, Xiao-Ru Huang ${ }^{2}$, Yong-Jiang Tang ${ }^{2}$, Xin-Yuan Guan ${ }^{5}$, Bryan Ping-Yen Yan², Ka-Fai To ${ }^{1}$ \\ \& Hui-Yao Lan ${ }^{1,2}$
}

TGF- $\beta$ is known to influence tumour progression. Here we report an additional role of Smad3 in the tumour microenvironment regulating cancer progression. Deletion or inhibition of Smad3 in the tumour microenvironment suppresses tumour growth, invasion and metastasis in two syngeneic mouse tumour models. Smad3 ${ }^{-1}-$ bone marrow gives rise to an expanded NK cell population with enhanced tumour-suppressive activities in vivo, and promotes differentiation of NK cells ex vivo. We identify E4BP4/NFIL3 as a direct Smad3 target gene critical for NK cell differentiation. Smad3 suppresses transcription of IFN- $\gamma$ via E4BP4 in a T-bet independent manner. Therefore disruption of Smad3 enhances both the E4BP4-mediated NK cell differentiation and anti-cancer effector functions in vivo and in vitro. Furthermore, systemic treatment with a Smad3 inhibitor SIS3 effectively suppresses cancer progression. In summary, suppression of NK cell-mediated immunosurveillance via the Smad3-E4BP4 axis contributes to cancer progression. We propose targeting Smad3-dependent tumour microenvironment may represent an effective anti-cancer strategy.

\footnotetext{
${ }^{1}$ Department of Anatomical and Cellular Pathology, The Chinese University of Hong Kong, Hong Kong SAR 999077, China. ${ }^{2}$ Li Ka Shing Institute of Health Sciences and Department of Medicine \& Therapeutics, The Chinese University of Hong Kong, Hong Kong SAR 999077, China. ${ }^{3}$ Clinical Translational Research Center, Shanghai Pulmonary Hospital, Shanghai 200092, China. ${ }^{4}$ Department of Histology and Embryology, Tongji University School of Medicine, Tongji University Cancer Institute, Shanghai 200092, China. ${ }^{5}$ Department of Clinical Oncology, The University of Hong Kong, Hong Kong SAR 999077 , China. * These authors contributed equally to this work. Correspondence and requests for materials should be addressed to H.Y.L. (email: hylan@cuhk.edu.hk).
} 
C ancer progression is dependent not only on the characteristics of cancer cells but also on the tumour microenvironment ${ }^{1,2}$. Transforming growth factor- $\beta$ (TGF- $\beta$ ) has been suggested to play a suppressive role in carcinogenesis, but paradoxically cancer cells also produce TGF- $\beta 1$ to support the tumour development ${ }^{3-6}$. Cancer cell-derived TGF- $\beta$ can promote tumour growth by triggering angiogenesis, epithelial-mesenchymal transition and the matrix metalloproteinase (MMP) system for ECM degradation ${ }^{3-6}$. There is an increasing evidence that pro-tumorigenic microenvironment is promoted by TGF- $\beta$ signalling ${ }^{7,8}$. Smad3 is a key mediator of canonical TGF- $\beta$ signalling pathway and plays an important role in the TGF- $\beta 1$-mediated transcriptional regulation ${ }^{9}$. Smad3-deficient mice exhibit impaired mucosal immunity and diminished T-cell responses to TGF- $\beta^{8,10}$. The lack of Smad3 resulted in severe graft-versus host disease by promoting Th1 differentiation and granulocyte-mediated tissue injury ${ }^{11}$. Smad3 can bind to the Foxp3 enhancer for the development of regulatory $\mathrm{T}$ (Treg) cells and differentially regulates the induction of Treg and Th17 immune responses ${ }^{12,13}$. Thus mice lacking Smad3 have impaired Treg development ${ }^{14}$. TGF- $\beta$ has a suppressive role in the cytolytic activity, especially on the interferon responsiveness of natural killer (NK) cells in vitro ${ }^{15,16}$. In addition, Trotta et al. ${ }^{17}$ elucidated that TGF- $\beta /$ Smad3 signalling can suppress CD16mediated interferon-gamma (IFN- $\gamma$ ) production in NK cells via reducing T-bet expression. Smad3 mutant mice were reported to develop metastatic colorectal cancer ${ }^{18}$, which may be related to chronic inflammation ${ }^{19,20}$. In contrast, mice lacking Smad3 were recently suggested to be resistant to chemical-induced skin carcinogenesis by $\mathrm{Li}$ et al. ${ }^{21}$. The discrepancy among the studies underlie the importance in further delineation of the mechanism of TGF- $\beta /$ Smad signalling in tumour progression. Nevertheless, these studies suggested that Smad3 could be an important checkpoint for TGF- $\beta$ signalling in the tumour microenvironment.

In the present study, we demonstrate the crucial role of Smad3-dependent tumour microenvironment in cancer progression by using two well-established syngeneic mouse tumour models. Mice lacking Smad3 are protected against tumour growth, invasion, metastasis and death. NK cell population in the $\mathrm{Smad} 3^{-1-}$ tumour microenvironment is dramatically expanded. This observation led us to identify that E4BP4 (Nfil3), one of the master transcription factors for NK cell differentiation $^{22-24}$, is a direct Smad3 target gene. Thus TGF- $\beta / S \operatorname{mad} 3$ signalling facilitates cancer progression by suppressing NK cell production via downregulating E4BP4. The crucial role of Smad3 is further supported by animal experiments with reconstitution of $\mathrm{GFP}^{+} \mathrm{Smad} 3^{-1-}$ bone marrow on $\mathrm{Smad} 3{ }^{+1+}$ mice and adoptive transfer of Smad3 ${ }^{-1-} \mathrm{NK} 1.1^{+}$cells into B16F10 tumour-bearing NOD/SCID mice. Furthermore, systemic treatment with a Smad3 inhibitor can suppress cancer progression by reversing the tumour promoting to an anticancer microenvironment in vivo. Therefore, our findings reveal the unexplored role of Smad3 in NK cell immunity and tumour microenvironment. The therapeutic potential of Smad3-targeted therapy for cancer treatment is also implicated.

\section{Results}

Smad3 is essential for cancer progression in mice. As TGF- $\beta$ / Smad signalling has been shown to be a pro-tumorigenic factor in cutaneous melanoma and lung cancer ${ }^{25,26}$, the role of Smad3 in tumour microenvironment was investigated by using two syngeneic mouse tumour models with luciferase-expressing invasive mouse lung carcinoma (LLC-luc) or melanoma (B16F10-luc) established in Smad3 ${ }^{-/}$(deletion of exon 8 and disruption of exon $7^{10}$ ) and their littermate $\operatorname{Smad} 3^{+/+}$mice (C57BL/6J background). During the first week after subcutaneous inoculation of LLC-luc cells, we observed almost equal tumour growth in both $\mathrm{Smad} 3^{-1-}$ and Smad3 $3^{+1+}$ mice. However, in the subsequent weeks, significant tumour regression was found in Smad3 $3^{-1-}$ mice where the tumours became undetectable by macroscopic and microscopic examinations and by bioluminescence imaging at week 3 (Fig. 1a-d). In contrast, the tumours in the Smad3 $3+$ mice grew rapidly and invasively, resulting in $40 \%$ mortality at week 3 (Fig. 1a-d). A similar pattern of cancer progression was observed in $\operatorname{Smad} 3^{-1-}$ and Smad $3^{+/+}$mice bearing a highly invasive melanoma cancer cell line B16F10 (Fig. 1e-g). In the B16F10 tumour-bearing Smad3 $3^{+/+}$mice, melanoma grew rapidly and invasively with metastasis to the lymph nodes, lung and colon, resulting in $60 \%$ mortality at week 3 (Fig. 1h and Supplementary Fig. 1). In contrast, these malignant features were absent in the tumour-bearing $\mathrm{Smad}^{-9-}$ mice in which survival rate was $100 \%$ without features of metastases (Fig. 1h and Supplementary Fig. 1). In addition, the protective effect of $\mathrm{Smad} 3^{-/}-$ microenvironment was further confirmed on a tumour rechallenging model (Supplementary Fig. 2A,B). These observations supported the importance of Smad3 in the tumour microenvironment for promoting tumour growth, invasion and metastasis. The supportive role of Smad3-dependent microenvironment in cancer metastasis was further examined in mouse metastatic models by intravenously (i.v.) administering LLC-luc or B16F10-luc cells into the mice. As shown in Fig. 1i-n, mice lacking Smad3 were resistant to cancer metastasis with barely detectable LLC-luc cancer cells and a few melanoma nodules in the lung. By contrast, Smad $3^{+/+}$mice developed massive lung cancer with a $100 \%$ mortality rate (Fig. 1o,p). These findings provided a clear and direct evidence for a crucial role of Smad3-dependent microenvironment in cancer progression.

Smad3 suppresses NK cell immunity in tumour-bearing mice. We next investigated the underlying mechanisms whereby mice lacking Smad3 were protected against cancer growth and metastasis. A 10 -fold increase in the number of tumourinfiltrating NK1.1 ${ }^{+} \mathrm{NKp} 46^{+}$and NK1.1 ${ }^{+}$IFN- $\gamma^{+} \mathrm{NK}$ cells was found in the B16F10 tumour-bearing mice lacking Smad3 compared with the Smad3 ${ }^{+I+}$ group (Fig. $2 \mathrm{a}-\mathrm{c}$ ). The inhibitory effect of Smad3-dependent microenvironment on $\mathrm{NK}$ cell population was further investigated. Native $\operatorname{Smad} 3^{-/-}$mice (without tumour inoculation) showed slight increase in mature $\mathrm{NK} 1.1^{+} \mathrm{CD}_{49 \mathrm{~b}}{ }^{+} \mathrm{NK}$ cells in the bone marrow, spleen, lung and peripheral blood compared with $\mathrm{Smad} 3^{+/+}$mice (Supplementary Fig. 3A). The population of NK1.1 ${ }^{+} \mathrm{CD} 49 \mathrm{~b}^{+}$ NK cells was further increased after cancer inoculation, resulting in a 10-fold increase in NK1.1 ${ }^{+} \mathrm{CD}_{4} 9 \mathrm{~b}^{+} \mathrm{NK}$ cells in the peripheral blood and lung and a 3 -fold increase in the spleen compared with the tumour-bearing $\mathrm{Smad} 3^{+1+}$ mice (Supplementary Fig.3B,C). The increase in mature NK1.1 ${ }^{+}$ NKp46 ${ }^{+} \mathrm{NK}$ cells was also observed in the tumour, spleen and blood of the B16F10-rechallenged mice lacking Smad3 (Supplementary Fig. 2C,D). In addition, deletion of Smad3 also enhanced the anticancer activities of NK cells by increasing the levels of granzyme B, interleukin (IL)-2 and IFN- $\gamma$ locally within the cancer microenvironment and in the systemic circulation (Fig. 2d,e). The splenic NK cells isolated from the B16F10 tumour-bearing Smad3 $3^{-1-}$ mice also exhibited a significant increase in the ex vivo cytotoxicity against tumour cells with higher levels of INF- $\gamma$ production when compared with the NK cells obtained from the tumour-bearing Smad3 ${ }^{+I+}$ mice (Supplementary Fig. 4). Moreover, a marked reduction in 

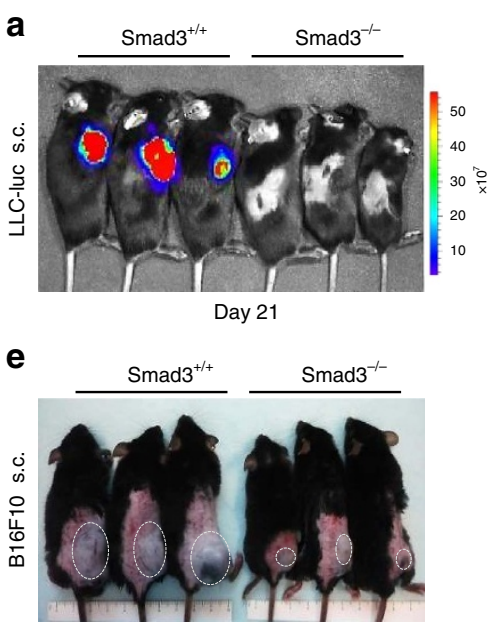

Day 21

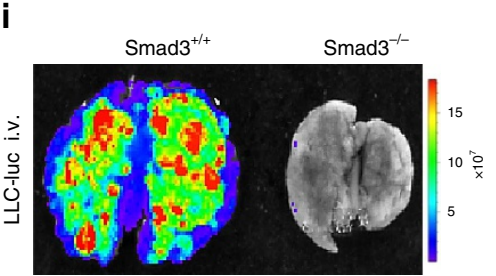

Day 21

I

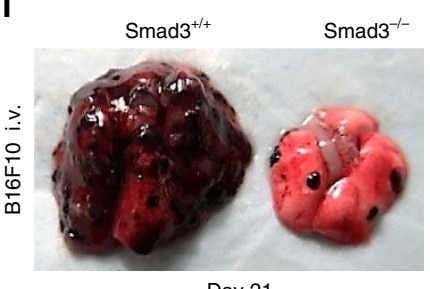

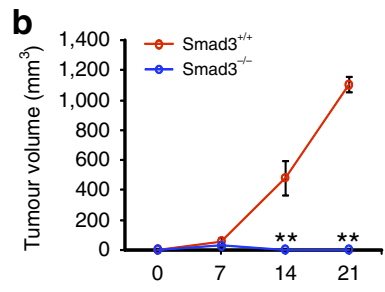

Days after LLC inoculation

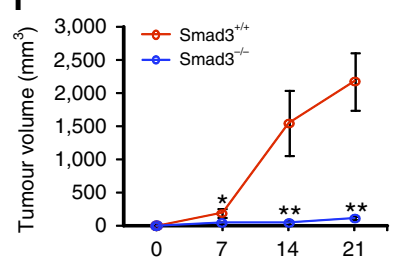

Days after B16F10 inoculation

j.

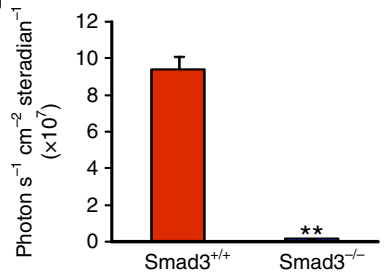

m

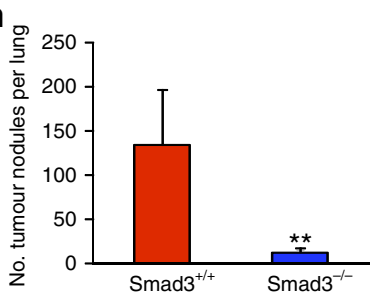

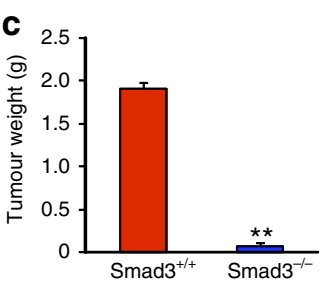

g

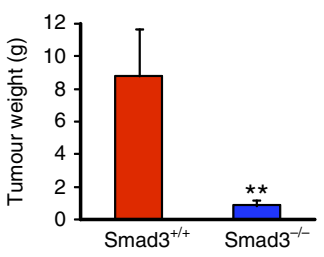

$\mathbf{k}$

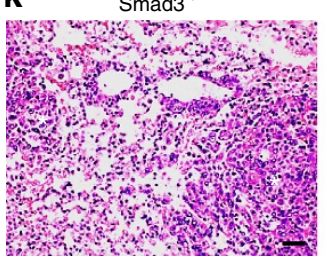

n $\quad \mathrm{Smad}^{+/+}$

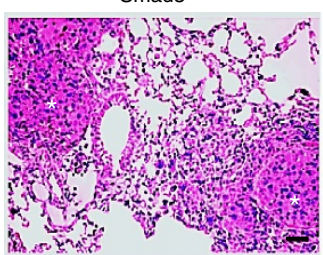

d

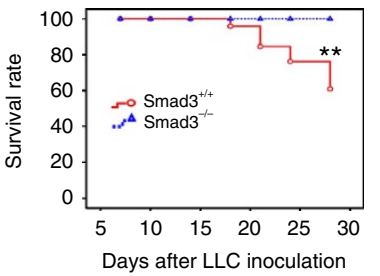

h

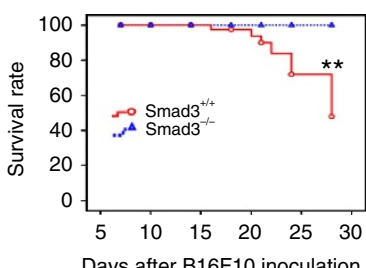

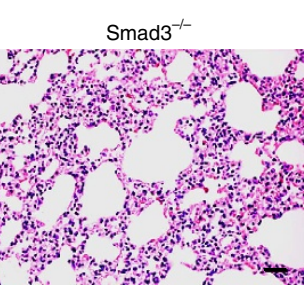

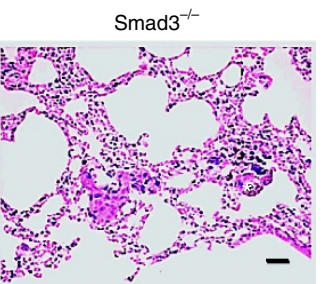

$\mathbf{0}$

\section{p}
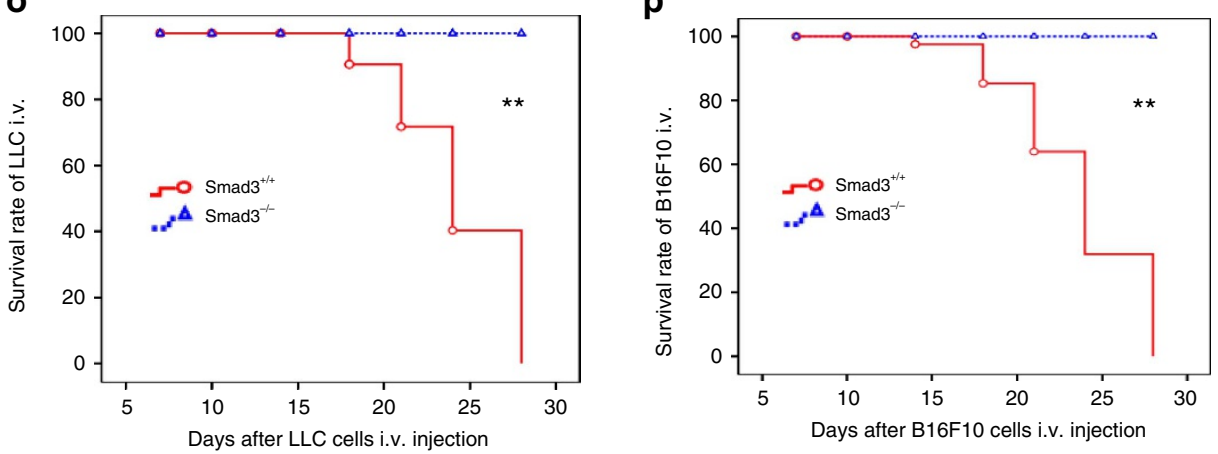

Figure 1 | Mice lacking Smad3 are protected from tumour progression. LLC-luc cancer cells were s.c. inoculated into Smad3 ${ }^{+/}+$and $\mathrm{Smad3} 3^{-/}-\mathrm{mice}$ $(n=3)$ and examined by bioluminescence imaging on day 21 (a), tumour volumes (b), tumour weights on day 21 (c) and the Kaplan-Meier plot of survival (d). B16F10 cells $\left(2 \times 10^{6}\right.$ cells per mouse) were s.c. inoculated into Smad3 ${ }^{+/+}$and Smad3 $-/-$mice $(n=5)$ and examined for the growth patterns (highlighted with white circles) on day 21 (e), tumour volumes (f), tumour weights on day 21 (g) and the Kaplan-Meier plot of survival (h). LLC-luc cells $\left(3 \times 10^{5}\right.$ cells per mouse) were i.v. inoculated into Smad3 $3^{+/}$and Smad3 $3^{-/-}$mice $(n=5)$ and examined for the metastatic growth patterns in the lung by bioluminescence imaging (i), quantitative photon intensities (j) and histology on H\&E-stained sections (k). B16F10 cells ( $3 \times 10^{5}$ cells per mouse) were i.v. inoculated in Smad3 ${ }^{+/+}$and Smad3 ${ }^{-/-}$mice $(n=5)$ and examined for metastatic growth in the lung by macroscopic morphology $(\mathbf{I})$, quantitative analysis of metastatic nodules (m) and histology on H\&E-stained sections (n). Note that the metastatic B16F10 tumours in the lung are shown by the dark-black nodules grossly and the metastatic LLC or B16F10 nodules on H\&E-stained sections are indicated with the white star ( ${ }^{\star}$ ). Kaplan-Meier plot of survival rates of metastatic LLC-luc (o) and B16F10 (p) tumour-bearing mice. Data represent the mean \pm s.d. for groups of mice as indicated in individual experiments above. ${ }^{\star} P<0.05,{ }^{\star \star} P<0.01$ compared with tumour-bearing Smad3 ${ }^{+/+}$mice analysed by analysis of variance. Scale bars, $50 \mu \mathrm{m}$.

vascular endothelial growth factor (VEGF) expression, CD31 ${ }^{+}$ blood vessels, $\mathrm{CD}^{+}{ }^{+}$Foxp3 ${ }^{+}$Treg cells and the expression of MMP-2, MMP-9, MMP-13 and C-X-C motif chemokine receptor 4 (CXCR4) in the tumour stroma were observed in the
Smad $3^{-1-}$ tumour microenvironment (Supplementary Figs 5 and 6). In contrast, depletion of NK cells from the tumourbearing hosts with a neutralizing antibody restored rapid progression of the B16F10 tumour only in Smad3 ${ }^{-/}$mice but not 
a
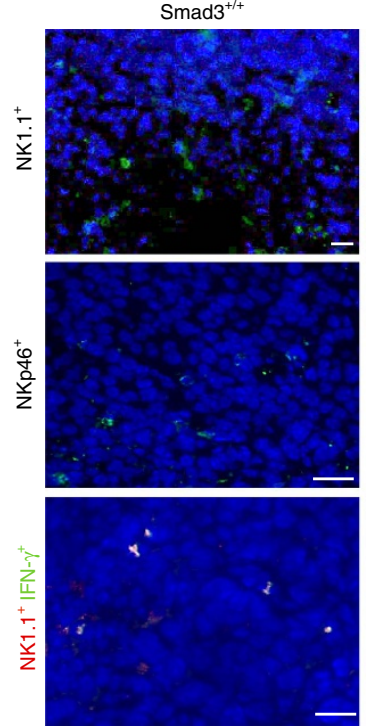

b
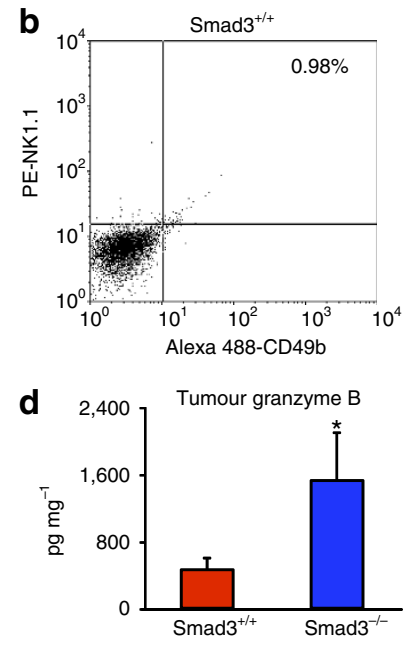

e

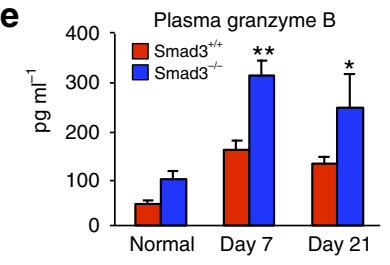

$\mathrm{Smad}^{-1-}$
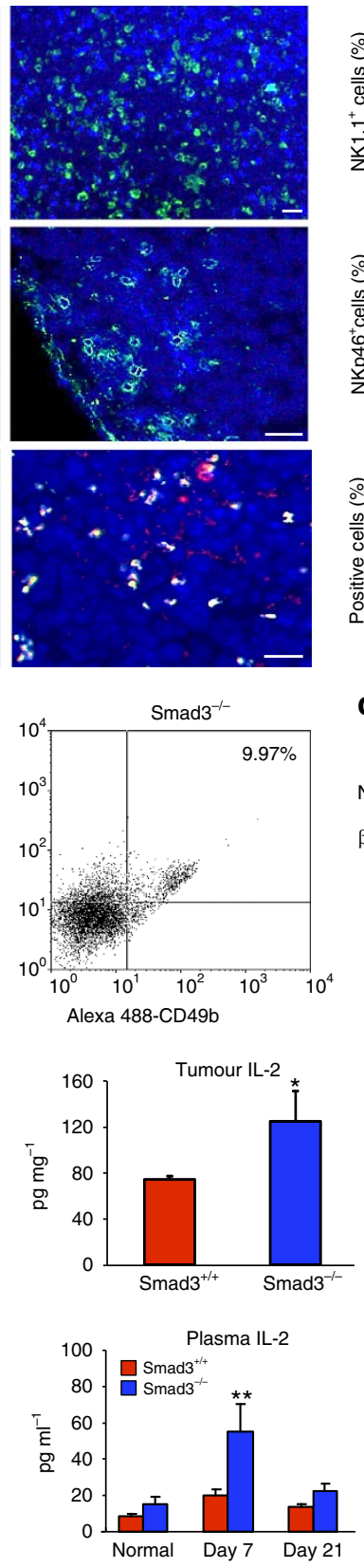
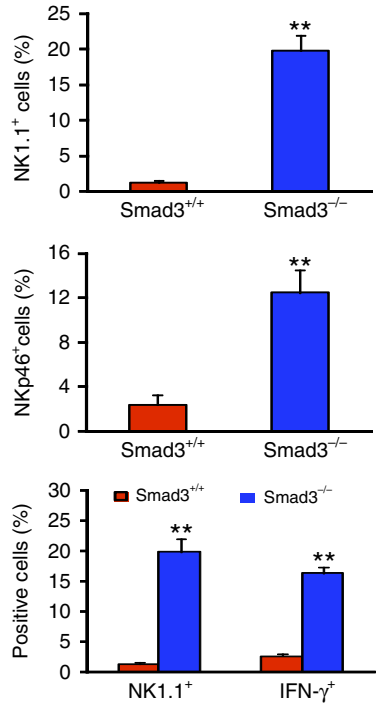

C
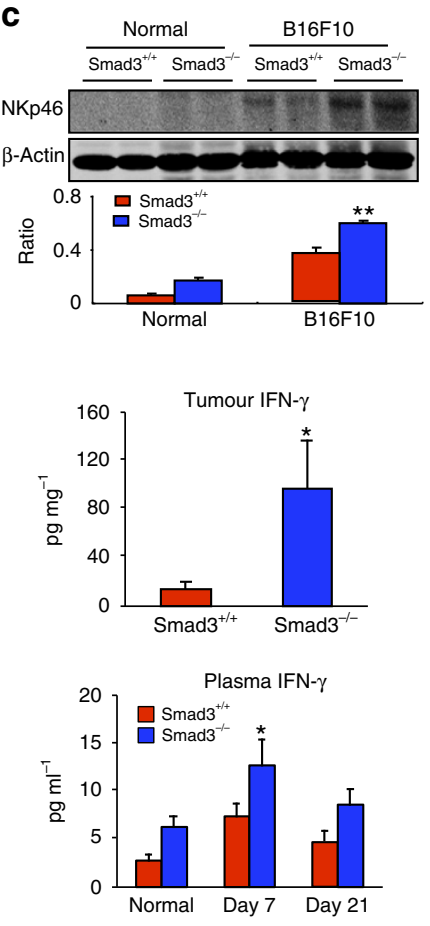

f
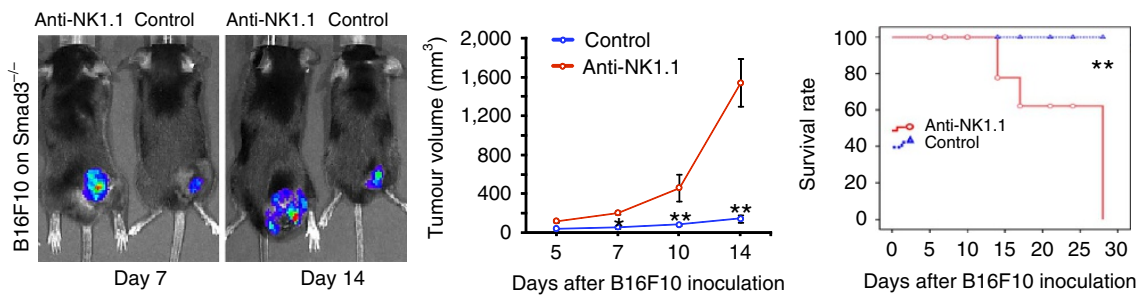

Days after B16F10 inoculation

Figure 2 | Smad3 facilitates cancer progression by suppressing host NK cell immunity in the tumour microenvironment. (a) Immunofluorescence detects the tumour-infiltrating NK1.1 ${ }^{+}, \mathrm{NKp} 46^{+}$and NK1.1 ${ }^{+}$INF- $\gamma^{+}$NK cells in the B16F10 tumour collected on day 7. Representative images of tumour sections stained with the antibodies recognizing NK1.1 (green, upper panel), NKp46 (green, middle panel), NK1.1 (red) and IFN- $\gamma$ (green, lower panel) are shown. Nuclei were counterstained with DAPI (blue), and the percentage of positive cells in the tumour tissues of Smad3 $3^{-/-}$or Smad3 ${ }^{+/+}$mice are shown (right panel). (b) Two-colour flow cytometry shows the population of tumour-infiltrating NK1.1 ${ }^{+}$CD49b ${ }^{+}$cells in the B16F10 tumour. (c) Western blotting analysis detects the NKp46 expression within the tumour tissues. (d,e) Enzyme-linked immunosorbent assay analysis determines the levels of granzyme B, IL-2 and IFN- $\gamma$ in the tumour tissues (d) and circulation (e). (f) Effects of NK cell depletion on cancer progression in B16F10 tumour-bearing Smad3 ${ }^{-/-}$mice as determined by bioluminescent imaging, tumour volume measurement and the survival rate. Data represent mean $\pm \mathrm{s}$.d. for groups of 3-5 mice. ${ }^{\star} P<0.05,{ }^{\star \star} P<0.01$ compared with B16F10 tumour-bearing Smad3 ${ }^{+/+}(\mathbf{a}-\mathbf{e})$ or Smad3 ${ }^{-/-}$mice (f) analysed by analysis of variance.

Scale bars, 50 (upper panel in a) and $100 \mu \mathrm{m}$. 
in Smad $3^{+1+}$ mice in vivo (Fig. $2 \mathrm{f}$ and Supplementary Fig. 7). These findings suggested an inhibitory role of Smad3 in NK cell development on a systemic level and a crucial role of NK cells in the Smad3-dependent tumour microenvironment.

Smad3 tumour microenvironment is derived from bone marrow. We then investigated the origin of Smad3-dependent tumour microenvironment by using $\mathrm{GFP}^{+} \mathrm{Smad}^{-/-}$and $\mathrm{GFP}^{+}$ Smad $3^{+I+}$ bone marrow chimeric mice bearing subcutaneous LLC-luc or B16F10-luc tumours. Our data showed that almost all stromal cells within the tumour tissue were derived from the transplanted bone marrow as recognized by their green fluorescent protein (GFP) expression (Fig. 3a). Compared with $\mathrm{GFP}^{+} \mathrm{Smad}^{+1+}$ chimeric mice, mice with $\mathrm{GFP}^{+} \mathrm{Smad}^{-1-}$ bone marrow exhibited significant reduction in the tumour growth and mortality rate (Fig. 3b,c), which was again associated with a 10 -fold increase in $\mathrm{GFP}^{+} \mathrm{NK}^{+} .1^{+}$cells in the tumour microenvironment (Fig. 3a). These results suggested that Smad3 may suppress host anticancer immunity at the bone marrow compartment. The inhibitory role of Smad3 on bone marrowderived NK cell immunity was confirmed in vitro; the cancerkilling activity of NK cells differentiated from Smad3 $-1-$ bone marrow cells was significantly higher than the Smad3 ${ }^{+} /+$control (Supplementary Fig. 8A). Interestingly, disruption of Smad3 (knockout or inhibition) facilitated NK cell maturation $\left(\mathrm{NKp} 46^{+}\right.$ IFN- $\gamma^{+}$cells) even in the presence of TGF- $\beta 1$ (Supplementary Fig. 8C). Therefore, deletion of Smad3 may enhance NK cell development in bone marrow and augmentation of host anticancer immunity at the distant tumour microenvironment.

Smad3 suppresses E4BP4-dependent NK cell maturation. As Smad3 ${ }^{-1}$ microenvironment was able to protect mice
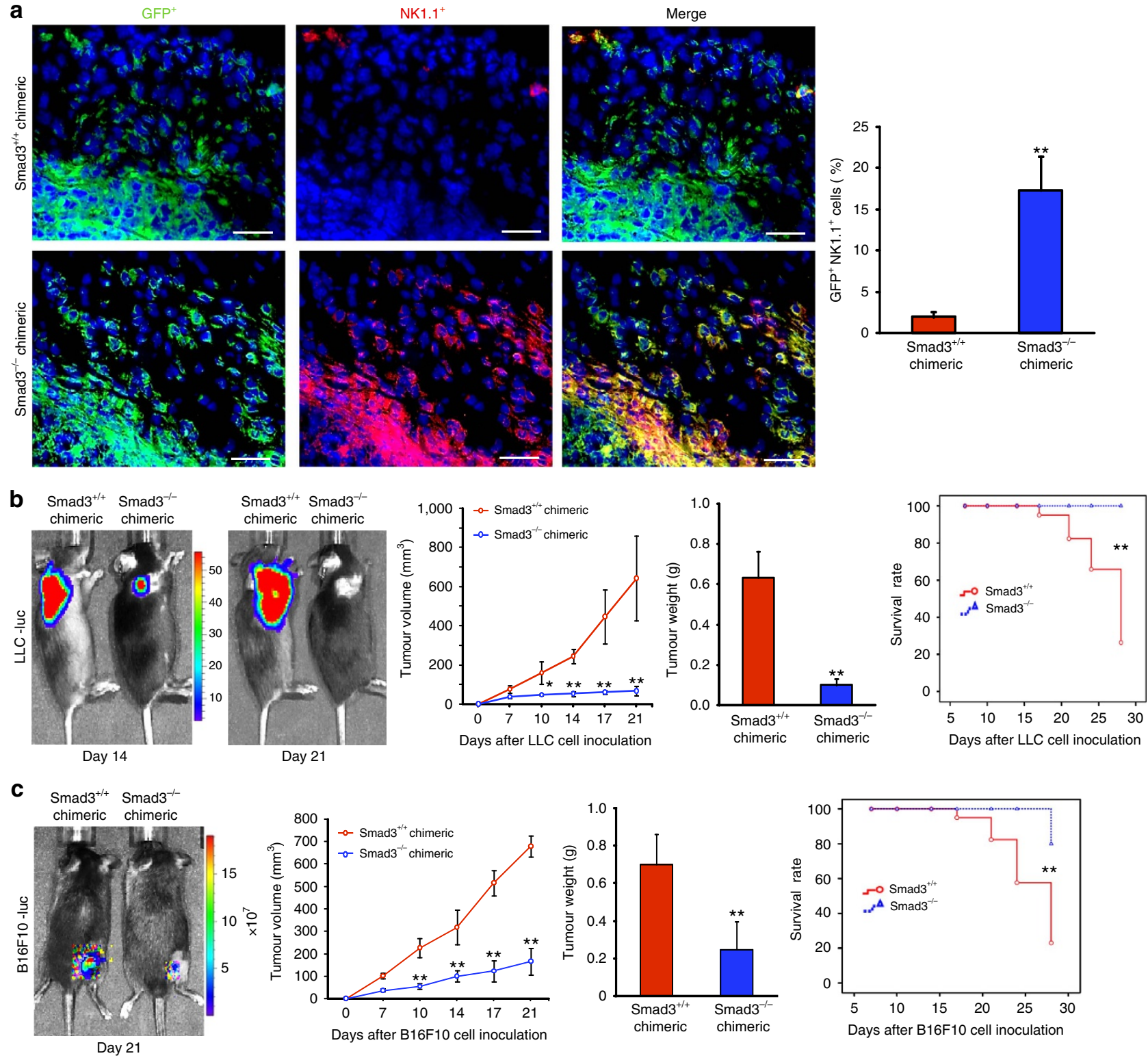

Figure 3 | Smad3-dependent tumour microenvironment is derived from the bone marrow. LLC-luc or B16F10-luc cancer cells were s.c. inoculated into $\mathrm{GFP}^{+}$Smad3 ${ }^{+/+}$and GFP + Smad3 ${ }^{-/-}$chimeric mice and examined for: (a) bone marrow-derived NK cells $\left(\mathrm{GFP}^{+} \mathrm{NK}_{1.1^{+}}\right)$in the tumour tissues by two-colour immunofluorescence; $(\mathbf{b}, \mathbf{c})$ tumour growth patterns by bioluminescent imaging, tumour volumes, tumour weights on day 21 and the survival rate of LLC $(\mathbf{b})$ or B16F10 (c) tumour-bearing chimeric mice. Data represent mean \pm s.d. for groups of $4-5$ mice. ${ }^{\star} P<0.05$, ${ }^{\star \star} P<0.01$ compared with tumour-bearing Smad3 ${ }^{+/+}$chimeric mice received GFP + Smad3 $^{-/-}$bone marrow transplantation analysed by analysis of variance. Scale bars, $100 \mu \mathrm{m}$. 
against cancer progression by dramatically increasing NK cell production (Figs 1-3 and Supplementary Figs 1 and 2), we further investigate the unexplored role of Smad3 as a negative regulator in the NK cell development. Western blottings showed that deletion of Smad3 increased the expression of E4BP4 (or Nfil3), an important transcription factor for NK lineage commitment ${ }^{22-}$ 24 , in both bone marrow-derived and splenic NK cells from the Smad $3^{-1-}$ mice on Day 10 after tumour inoculation (Fig. 4a). It suggested a close link between Smad3 and NK cell development in the tumour microenvironment. To examine the inhibitory role of TGF- $\beta / \mathrm{Smad} 3$ signalling in E4BP4, total bone marrow cells or untouched splenic NK cells from Smad3 ${ }^{+1+}$ or Smad3 $3^{-1-}$ mice were stimulated with TGF- $\beta 1$ for $3 \mathrm{~h}$ and subjected for realtime PCR analysis. As shown in Fig. $4 \mathrm{~b}-\mathrm{d}$, TGF- $\beta 1$ significantly suppressed the transcription of E4BP4 mRNA in Smad3 ${ }^{+1+}$ bone marrow cells and splenic NK cells, which was blunted when Smad3 was genetically deleted or pharmacologically inhibited with Smad3 inhibitor (SIS3). We next investigated the functional importance of E4BP4 in Smad3-mediated suppression of NK cell development by transfecting bone marrow cells from Smad $3+1+$ and Smad $3^{-1}$ mice with nonsense (control) or short interfering RNA (siRNA) against mouse E4BP4 (siE4BP4) and cultured under NK cell differentiation medium for 6 days. Real-time PCR detected that SIS3 treatment increased E4BP4 mRNA expression in Smad3 $3^{+1+} \mathrm{NK}$ cells, whereas transient knockdown with siE4BP4 resulted in significant inhibition of E4BP4 mRNA expression in bone marrow-derived $\mathrm{Smad} 3^{+1+}$ and $\mathrm{Smad} 3^{-1-}$ NK cells as well as SIS3-treated Smad3 ${ }^{+1+}$ NK cells (Supplementary Fig. 9). Strikingly, while the production of $\mathrm{CD} 244^{+} \mathrm{NKp}^{+} 6^{+}$(immature NK cells) and CD244 ${ }^{+} \mathrm{NKp} 46^{-}$ (NK cell progenitors) cells was significantly accelerated in Smad $3^{-l-}$ or SIS3-treated Smad3 ${ }^{+l+}$ bone marrow cells, this promoting effect was diminished when E4BP4 was knocked down (Fig. 4e,f), demonstrating the functional importance of E4BP4 in the Smad3-mediated NK cell differentiation. Furthermore, a conversed Smad3-binding site (SBS) was found within the $3^{\prime}$ UTR (untranslated region) of E4BP4 gene (Fig. 4g) by using ECR browser (rVista 2.0, http://rvista.dcode.org/) 27 . The physical binding of Smad3 on the UTR of E4BP4 gene was enriched by TGF- $\beta 1$ stimulation as shown by the results of chromatin immunoprecipitation (ChIP) assay (Fig. 4h,i). Thus Smad3 transcriptionally inhibited E4BP4 in response to TGF- $\beta 1$, which was reversed when $\mathrm{Smad} 3$ is mutated as demonstrated by the luciferase reporter assay (Fig. 4j). These findings revealed the inhibitory role of Smad3 in NK cell development via downregulating the transcription of its direct target gene E4BP4. Given that deficiency of Smad3 prevents TGF- $\beta 1$ mediated inhibition of E4BP4 expression, targeting the TGF- $\beta 1 /$ Smad3-E4BP4 axis to accelerate the development of NK cells may enhance their anticancer immunity within the tumour microenvironment.

TGF- $\beta 1$ suppresses NK cell immunity via Smad3-E4BP4 axis. The specific role of Smad3 on NK cell-mediated antitumour activities was further examined by adoptive transfer of Smad3 ${ }^{+I+}$ or Smad3 ${ }^{-I-}$ NK1.1 ${ }^{+}$cells into the B16F10 tumour-bearing NOD/SCID mice in which the $\mathrm{NK}$ cell population was deficient ${ }^{28}$. Noticeably, NOD/SCID mice that received Smad3 ${ }^{-1-}$ NK cells showed a greater inhibition of tumour growth compared with those that received Smad3 $3^{+1+}$ NK cells (Fig. 5a,b). Trotta et al. ${ }^{17}$ recently reported of suppressed CD16-mediated IFN- $\gamma$ production of NK cells by TGF- $\beta 1$ via Smad3-T-bet-dependent pathway, so we compared the relative impact of E4BP4 versus T-bet on Smad3-mediated NK cell antitumour activities by adoptive transfer of the Smad3 $3^{-/-} \mathrm{NK}$ cells with or without knockdown of E4BP4 (Nfil3) or T-bet into the B16F10 tumour-bearing NOD/SCID mice. Interestingly, mice that received E4BP4 knockdown Smad3 ${ }^{-/-}$NK cells (siE4BP4) exhibited a higher tumour growth rate, which was associated with lower levels of infiltrating $\mathrm{NK} 1.1^{+} \mathrm{CD} 3{ }^{-} \mathrm{NK}$ cells and the expression of NKp46 when compared with those that received siT-bet Smad3 $3^{-I}-$ NK cells (Fig. $5 c, d$ ). In vitro study also confirmed this observation that NK differentiation and IFN- $\gamma$ expression were more significantly inhibited by knockdown of E4BP4 compared with that in T-bet knockdown Smad $3^{-1-}$ NK cells (Fig. 5e). A direct E4BP4-binding site on the promoter of IFN- $\gamma$ (which is $208 \mathrm{nt}$ apart from the T-bet-binding site) is predicted by ECR browser and therefore the results supporting that knockdown of E4BP4 suppressed IFN- $\gamma$ expression in a T-bet-independent manner (Supplementary Fig. 10).

Targeting Smad3 protects against cancer progression. The encouraging findings from tumour-bearing Smad3 ${ }^{-1-}$ mice leads us to further test a hypothesis that targeting Smad3dependent tumour microenvironment may protect mice against cancer progression. This was examined on Smad3 ${ }^{+1+}$ mice bearing B16F10 or LLC tumours by inactivating Smad3 signalling with an inhibitor SIS3 that specifically blocks the phosphorylation and DNA binding of Smad3 proteins ${ }^{29}$. The systemic treatment of SIS3 significantly inhibited the phosphorylation of Smad3 in both LLC and B16F10 tumour tissues and suppressed cancer progression in a dosage-dependent manner, resulting in a $100 \%$ survival rate (Figs $6 \mathrm{a}-\mathrm{d}$ and $7 \mathrm{a}$ and Supplementary Figs $11 \mathrm{~A}-\mathrm{D}$ and 12A). More importantly, SIS3 treatment significantly increased NK cell production in a dose-dependent manner, showing up to a fivefold increase in $\mathrm{NKp} 46^{+}$cells in tumour tissues (Fig. 6e). Inhibition of Smad3 also enhanced anticancer activities of NK cells by increasing releases of granzyme B, IL-2 and IFN- $\gamma$ locally within the tumour tissues and systemically in the circulation (Fig. 6f,g). Depletion of NK cells from SIS3-treated B16F10 tumour-bearing mice partially reversed the antitumour effects of SIS3 (Supplementary Fig. 13), which further supports the promoting role of Smad3-mediated NK suppression in cancer progression. In vitro study also confirmed this finding that pharmacological inhibition of Smad3 signalling with a SIS3 was capable of enhancing cancer-killing activities in both bone marrow-derived or splenic NK cells (Supplementary Fig. 8A,B). We demonstrated that the enhanced NK cell-mediated anticancer immunity has an important role in the anticancer effects of Smad3-dependent tumour microenvironment targeted treatment. Furthermore, systemic treatment of SIS3 also significantly altered the tumour-friendly microenvironment, including suppression on angiogenesis (VEGF expression and $\mathrm{CD}_{3}{ }^{+}$vessels) and tumour-invasive factors (MMP-2, MMP-9, MMP-13 and CXCR4) (Fig. 7b-d, Supplementary Fig. 12B-D). In vitro treatment with SIS3 was also able to inhibit the proliferation of B16F10 melanoma cells in a dose-dependent manner (Supplementary Fig. 14) and this may also suggest a direct inhibitory effect of SIS3 on tumour cell growth. Taken together, our results revealed that targeting Smad3-dependent microenvironment may represent a novel and effective therapy for invasive cancer.

\section{Discussion}

TGF- $\beta$ has a supportive role in progression of established tumour and Smad3 is a key mediator in the canonical TGF- $\beta$ signalling pathway. However, the potential role of Smad3 in the TGF- $\beta 1$ dependent tumour microenvironment remains incompletely characterized. In this study, we demonstrated that Smad3 is 
a

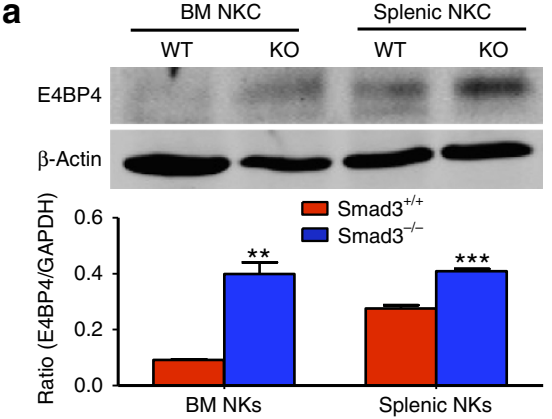

b

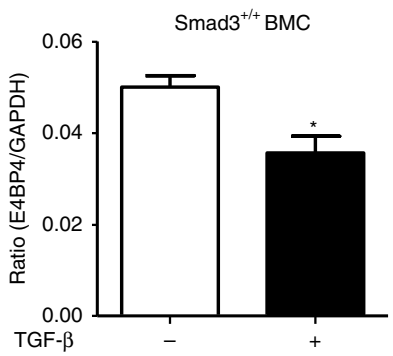

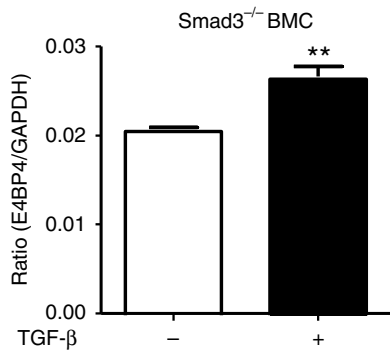

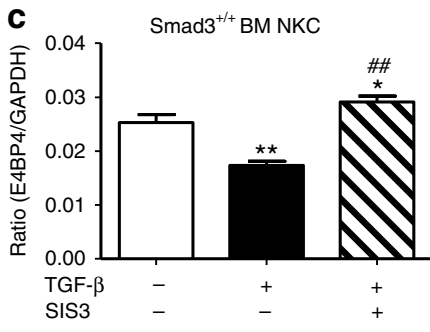

e
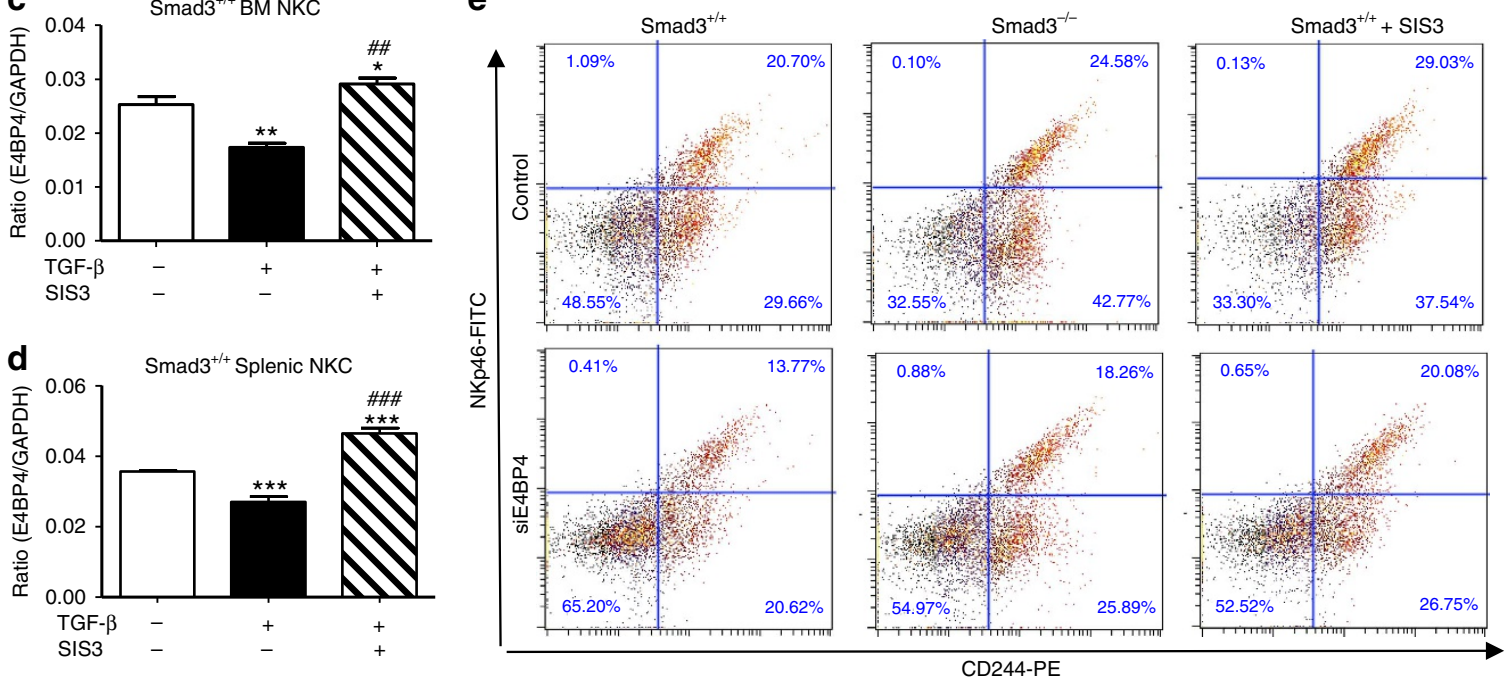

f

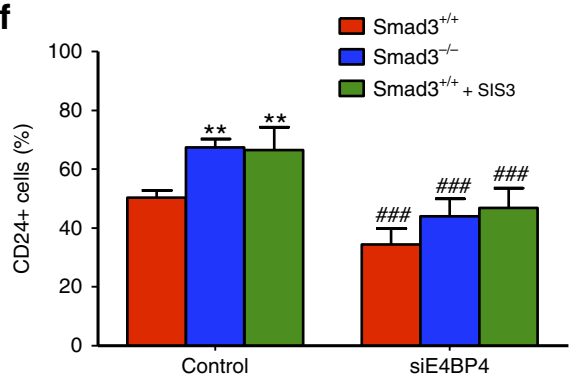

g

Smad3-binding site on UTR of E4BP4

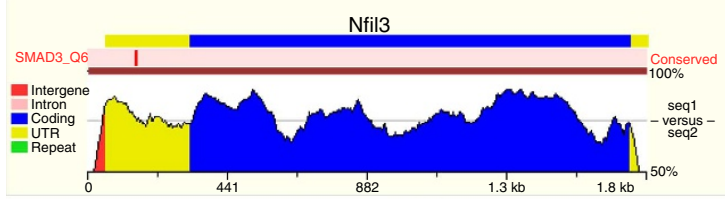

Mouse CTCTGTGGGGGGCTATCTGACTACACGCCAGGCA chr13:52967291

$\|::|:::||:|||||||||||:||:|||||| \mid$

Human CTTCGCATGGA-CTATCTGACTATACAC-AGGCA chr9:94171215

h
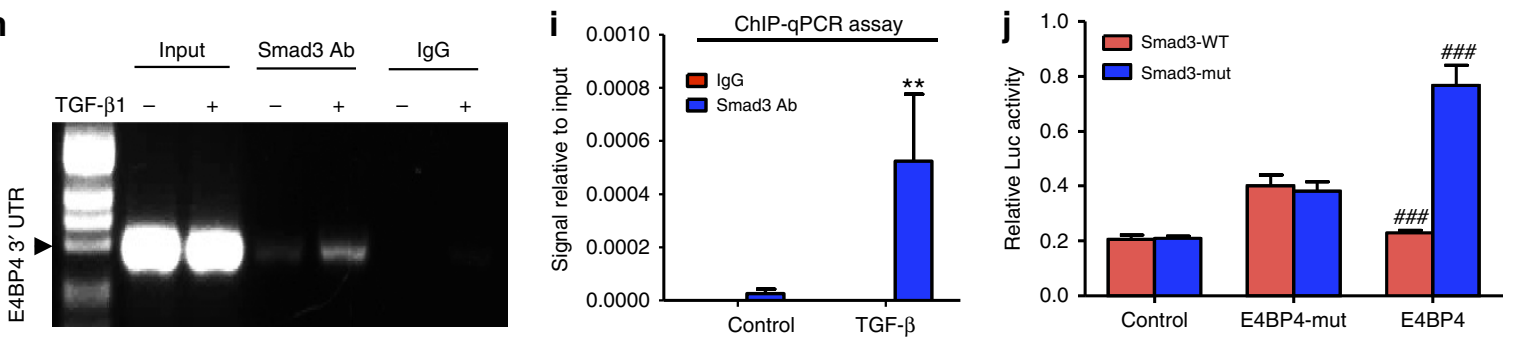

Figure 4 | Smad3 suppresses NK cell differentiation by downregulating E4BP4 in vivo and in vitro. (a) Western blotting analysis shows the expression of E4BP4 in the NK cells isolated from the bone marrow and spleen of the B16F10 tumour-bearing Smad3 ${ }^{+/+}$and Smad3 $3^{-/-}$mice on day 10 after tumour inoculation. (b) Real-time PCR detects the effect of TGF- $\beta 1\left(1 \mathrm{ng} \mathrm{ml}{ }^{-1}\right)$ on the transcription of E4BP4 in Smad3 ${ }^{+/+}$and Smad3 ${ }^{-/-}$bone marrow cells at $3 \mathrm{~h}$. (c,d) Effect of SIS3 $(2 \mu \mathrm{M})$ on TGF- $\beta 1$-mediated E4BP4 mRNA expression in bone marrow or spleen-derived NK cells. (e) Two-color flow cytometry detects the effects of knockdown of E4BP4 and deletion (Smad3 ${ }^{-/-}$) or inhibition (SIS3) of Smad3 in the production of bone-marrow cell derived $\mathrm{CD} 244^{+} \mathrm{NKp} 46^{-}$(NK progenitor) and CD244+ ${ }^{+} \mathrm{NKp} 46^{+}$(immature NK) cells (upper panels) on Day 6. (f) Quantitative results of CD244 ${ }^{+}$ cells from flow cytometry analysis. (g) A predicted SBS at the $3^{\prime}$ UTR of the evolutionarily conserved region of E4BP4 in human and mouse genomes (upper panel), indicated by bold and underlined (lower panel). (h,i $)$ ChIP assay was performed with Smad3 $+/+$ bone marrow cells stimulated with TGF- $\beta 1\left(1 \mathrm{ng} \mathrm{ml}^{-1}\right)$ and the enrichment of Smad3 binding to the $3^{\prime}$ UTR of E4BP4 gene was qualified by PCR (h) and quantified by real-time PCR (i). (j) Dual luciferase assay shows the inhibitory effect of Smad3 binding on E4BP4 3' UTR reporter activity. Data represent mean \pm s.e.m. for three independent experiments, ${ }^{\star} P<0.05,{ }^{\star \star} P<0.01,{ }^{\star \star \star} P<0.001$ compared with the control group $(\mathbf{b}-\mathbf{d}, \mathbf{i})$ or Smad3 ${ }^{+/+}$cells $(\mathbf{a}, \mathbf{f})$; ${ }^{\#} P<0.05,{ }^{\# \#} P<0.01$, $\# \# P<0.001$ compared with the TGF- $\beta 1$-treated group $(\mathbf{c}, \mathbf{d})$, individual controls (f) or E4BP4-mutant (j) analysed by analysis of variance. 

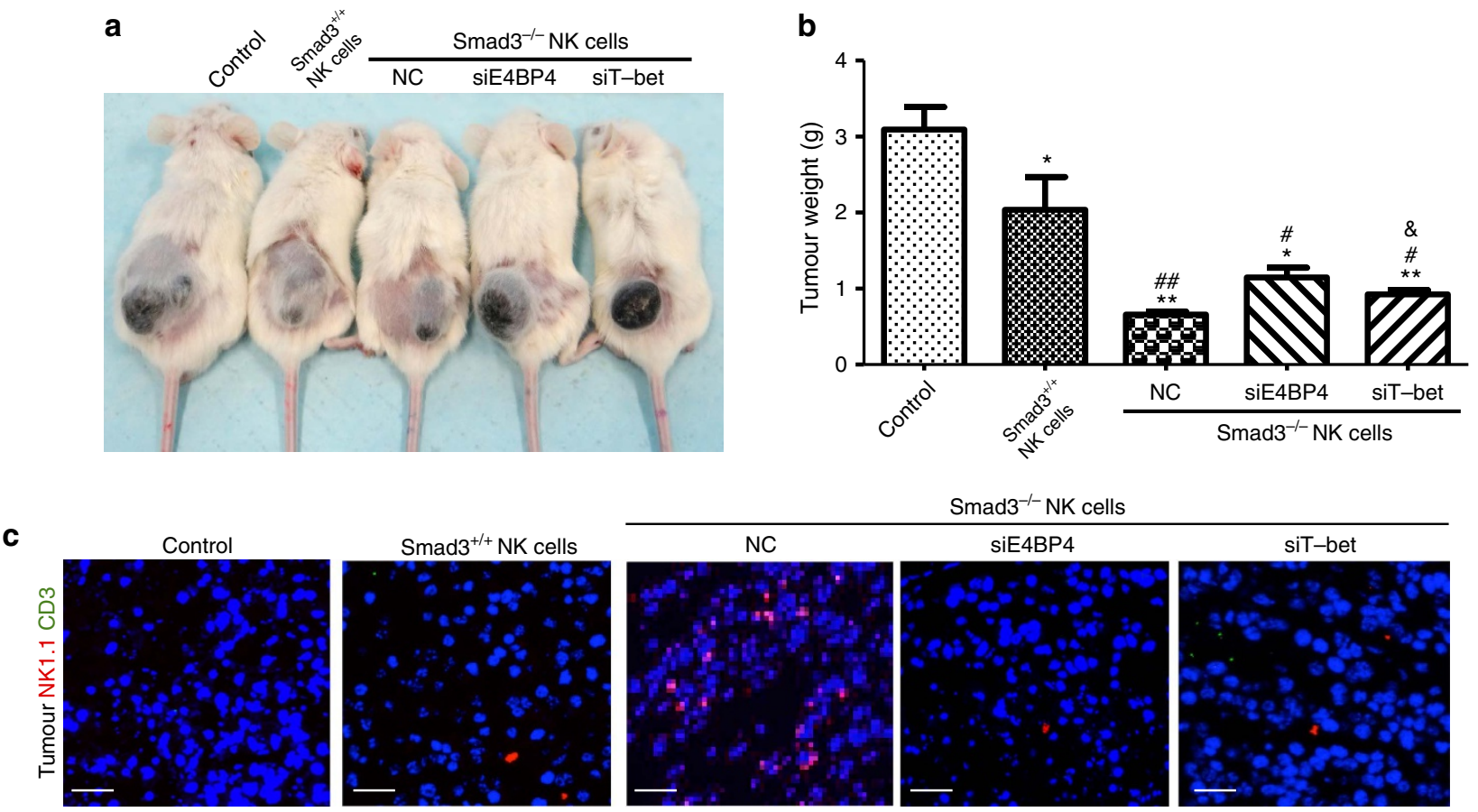

Smad3 ${ }^{-/-}$NK cells

d
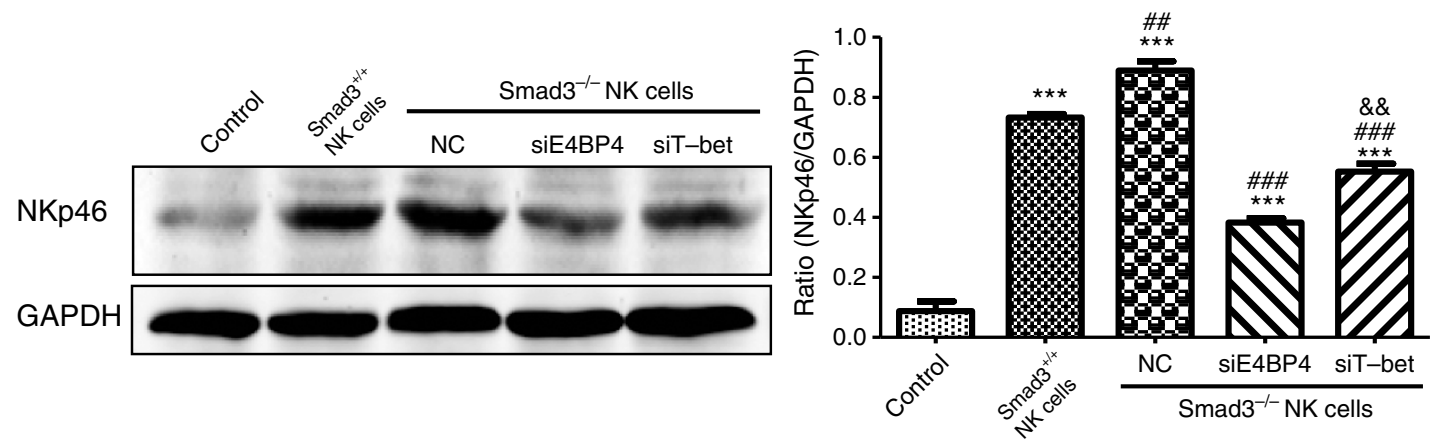

$\mathbf{e}$
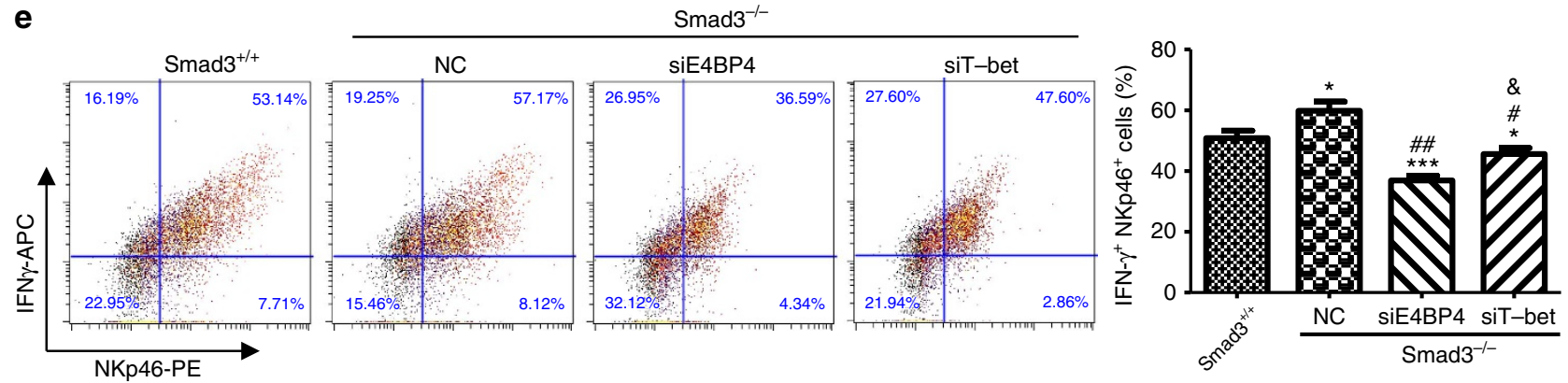

Figure 5 | The anticancer effect of Smad3 ${ }^{-/-}$NK cells is dependent on E4BP4 more than on T-bet. (a) Saline (Control), nonsense-treated Smad3 $+/+$ $\left(S_{3} 3^{+/+} \mathrm{NK}\right)$, nonsense-treated Smad3 ${ }^{-/-}$(NC) NK cells or Smad3 ${ }^{-/-}$NK cells with E4BP4 knockdown (siE4BP4) or T-bet knockdown (siT-bet) were infused (i.v.) into B16F10 tumour-bearing NOD/SCID mice and the antitumour effects are qualified by imaging on day 10 after NK cell infusion.

(b) Tumour weight after NK cell infusion on day 10. (c) Tumour-associated NK cells detected by two-colour immunofluorescence with the anti-NK1.1 and anti-CD3 antibodies (scale bars, $100 \mu \mathrm{m})$. Note that most of anti-NK1.1 ${ }^{+}$cells within the tumour microenvironment are negative for CD3. (d,e) Effect of E4BP4 and T-bet on ex vivo NK differentiation in Smad3 ${ }^{+/+}$or Smad3 $3^{-/-}$bone marrow cells on day 7. Bone marrow cells were transfected with siE4BP4 or siT-bet and the NK cell population in B16F10 tumour was detected by western blotting with NKp46 (d) or by two-colour flow cytometry with the antiNKp46 and IFN- $\gamma$ antibodies (e). Data represent mean \pm s.e.m. for groups of three mice or at least three independent experiments. ${ }^{\star} P<0.05$, ${ }^{\star \star} P<0.01$, ${ }^{\star \star \star} P<0.001$ compared with the control group $(\mathbf{b}, \mathbf{d})$ or Smad3 $+/+$ NK cells $(\mathbf{e}) ;{ }^{\#} P<0.05,{ }^{\# \#} P<0.01,{ }^{\# \# \#} P<0.001$ compared with the S3WT-NK group; $\& P<0.05$ and $\& \& P<0.01$ compared with the siE4BP4 group analysed by analysis of variance.

essential for TGF- $\beta 1$-mediated cancer progression, as both genetic deletion and pharmacological inhibition of Smad3 produced a significant inhibition of cancer growth, invasion and metastasis, resulting in a markedly improved survival rate in mouse models of LLC lung cancer and B16F10 melanoma.
Increase in production and anticancer activities of NK cells but decrease in angiogenesis, Treg response and tumour-invasive activities were found in the Smad3-knockout or -inhibited tumour-bearing mice. It is suggested that TGF- $\beta 1$-mediated pro-tumorigenic microenvironment can be shifted into a 
a

SIS3 treatment $\left(\mu \mathrm{g} \mathrm{g}^{-1}\right)$
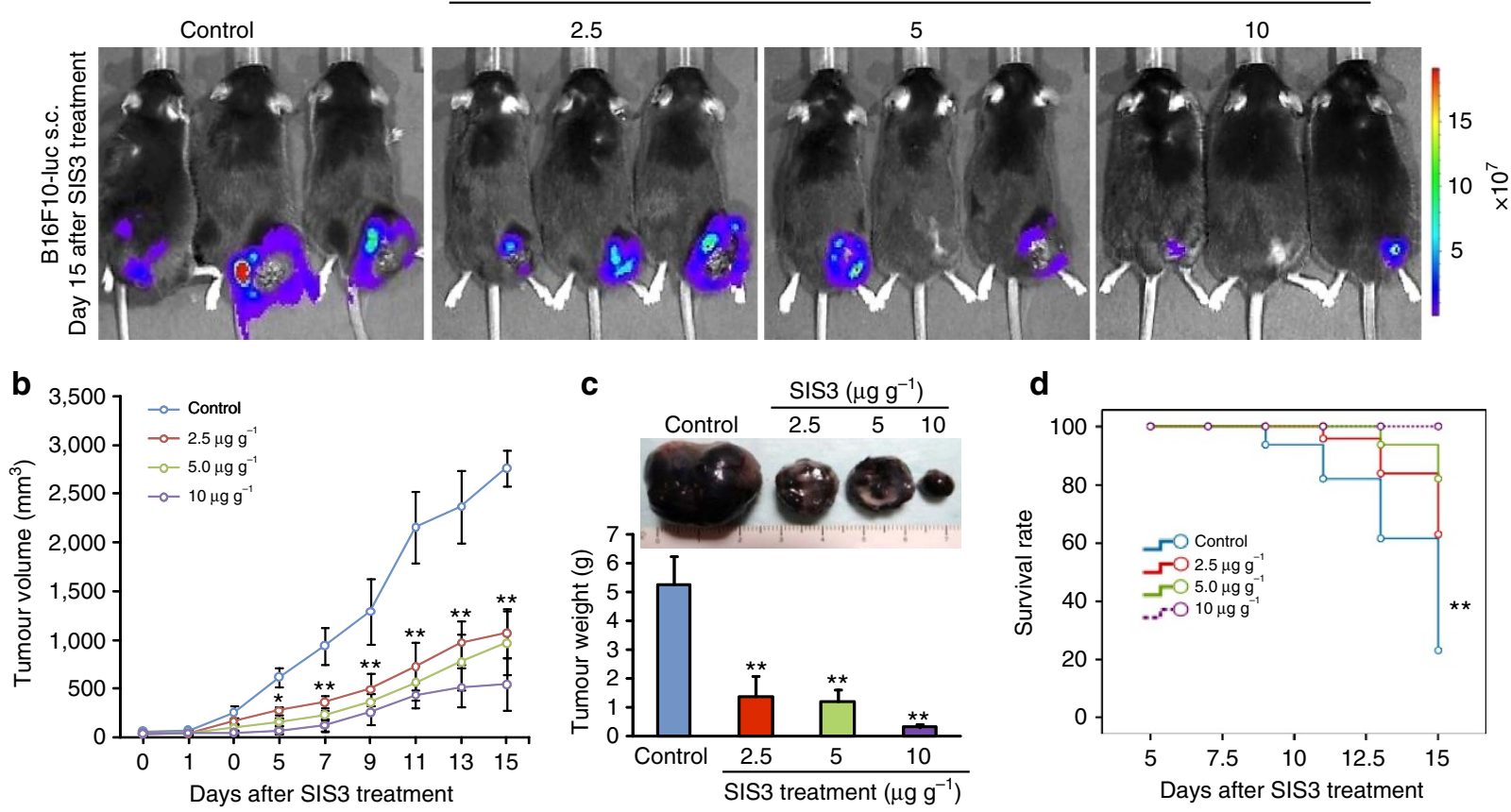

e
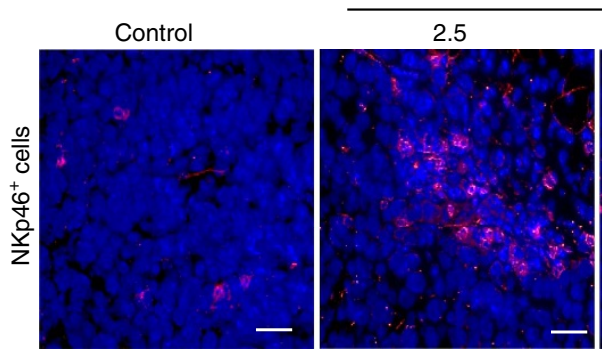

SIS3 treatment $\left(\mu \mathrm{g} \mathrm{g}^{-1}\right)$

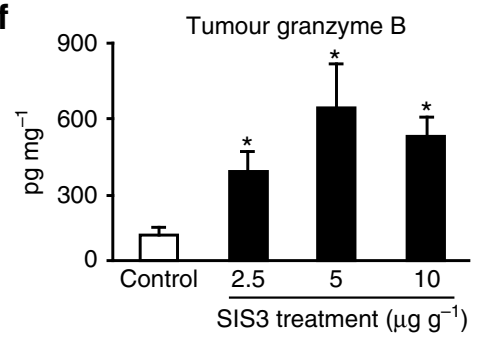

g

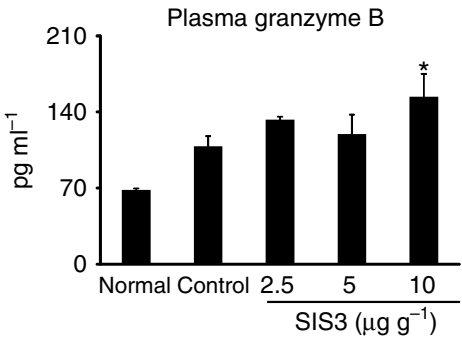

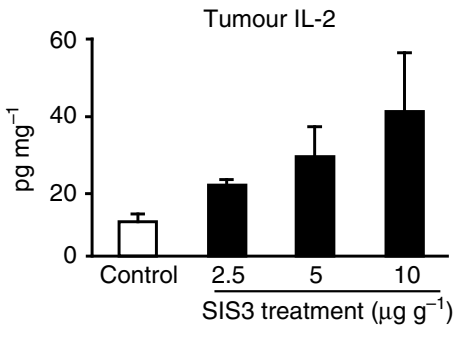

Plasma IL-2

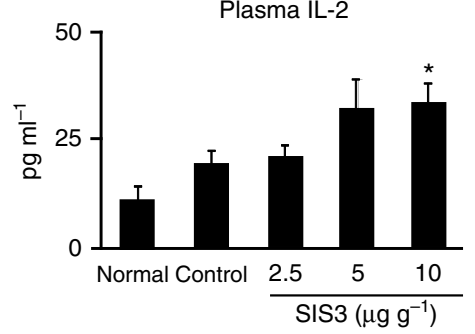

10
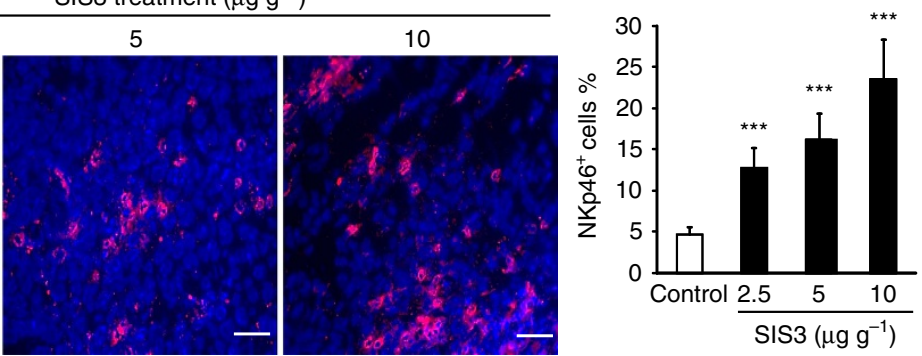

Tumour IFN- $\gamma$

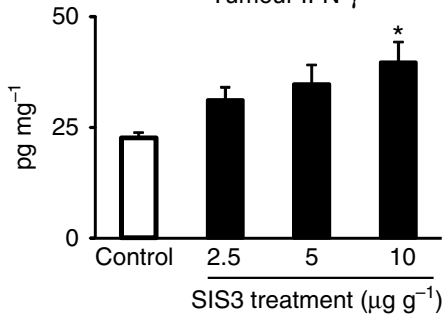

Plasma IFN- $\gamma$

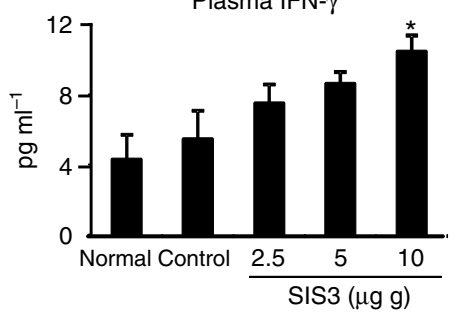

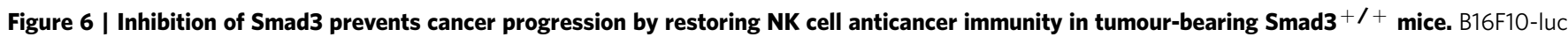
cancer cells were s.c. inoculated into Smad3 ${ }^{+/+}$mice and followed by treatment with various dosages of SIS3 $\left(0,2.5,5.0\right.$ or $10 \mu g g^{-1}$ day $^{-1}$, i.p.). (a) Representative bioluminescent images of the B16F10-luc tumours, (b) tumour volumes, (c) tumour weights on day 15 after treatment, (d) survival rates and (e) representative immunofluorescent images of tumour sections stained with the anti-NKp46 antibody (red) on day 15 after treatment. Nuclei were counterstained with DAPI (blue). (f,g) Enzyme-linked immunosorbent assay detects the levels of granzyme B, IL-2 and IFN- $\gamma$ in the tumour tissues (f) and circulation (g) on day 15 after SIS3 treatment. Data represent mean \pm s.d. for groups of $4-6$ mice. ${ }^{\star} P<0.05,{ }^{\star \star} P<0.01,{ }^{\star \star \star} P<0.001$ compared with control (saline-treated) analysed by analysis of variance. Scale bars, $100 \mu \mathrm{m}$. 

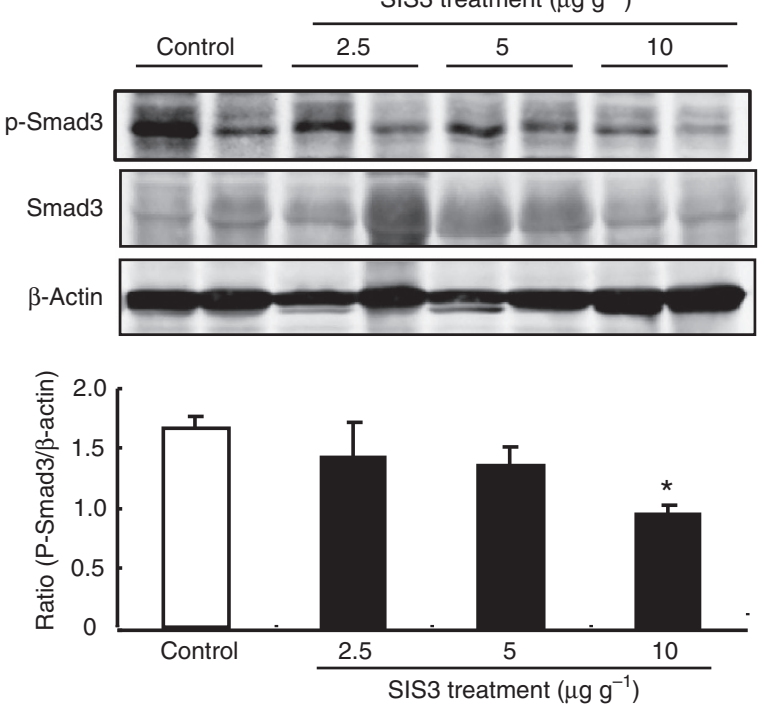

b
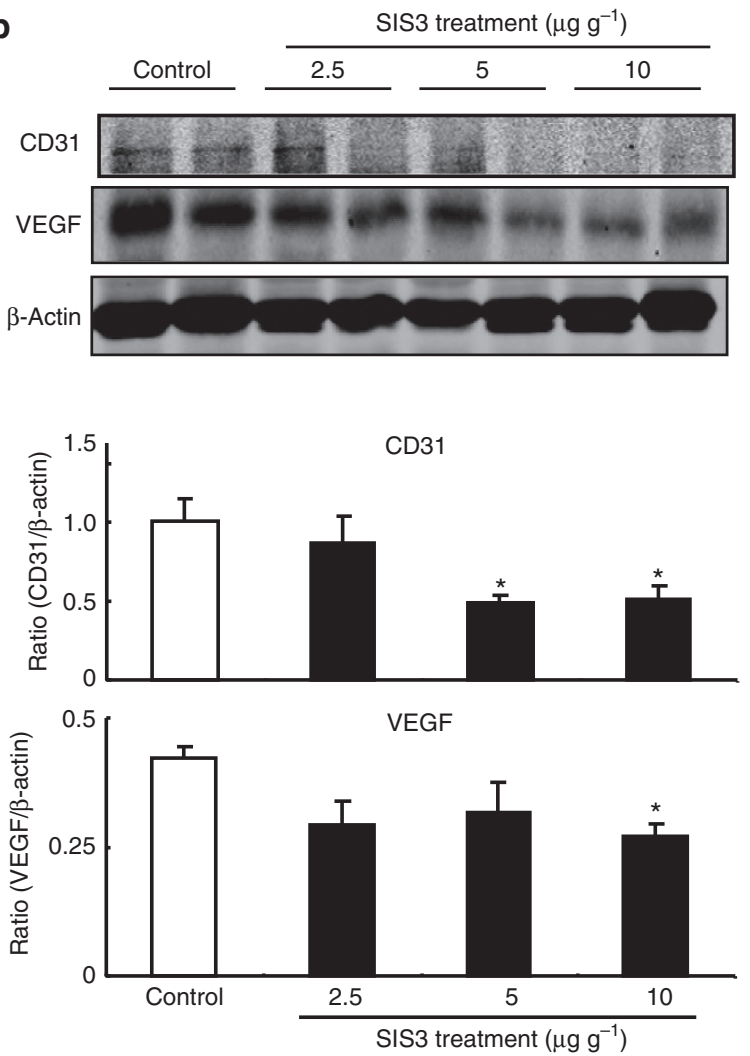

C

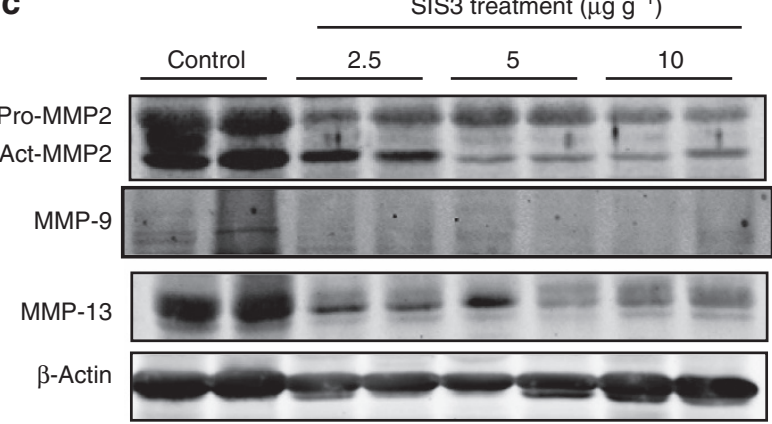

MMP-2
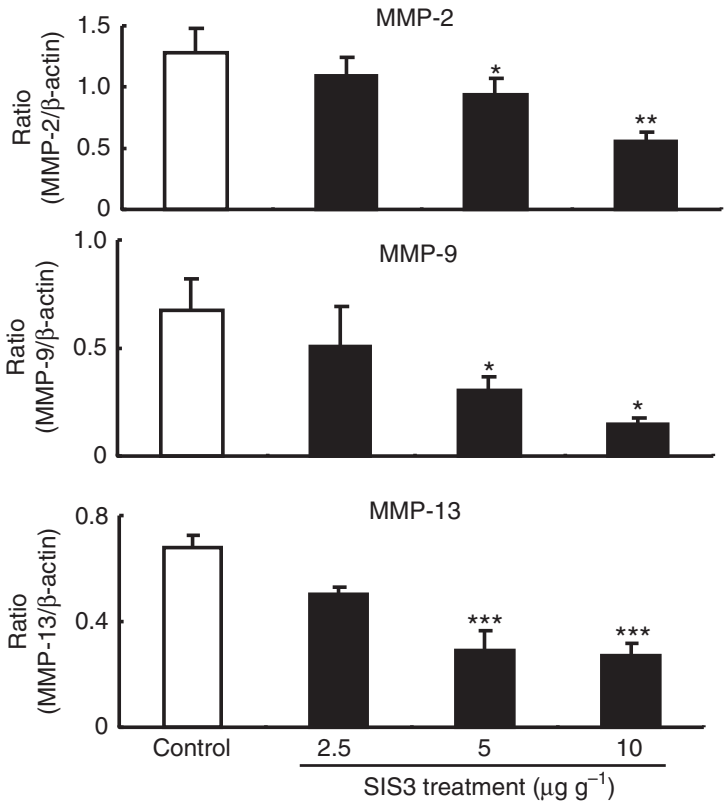

d
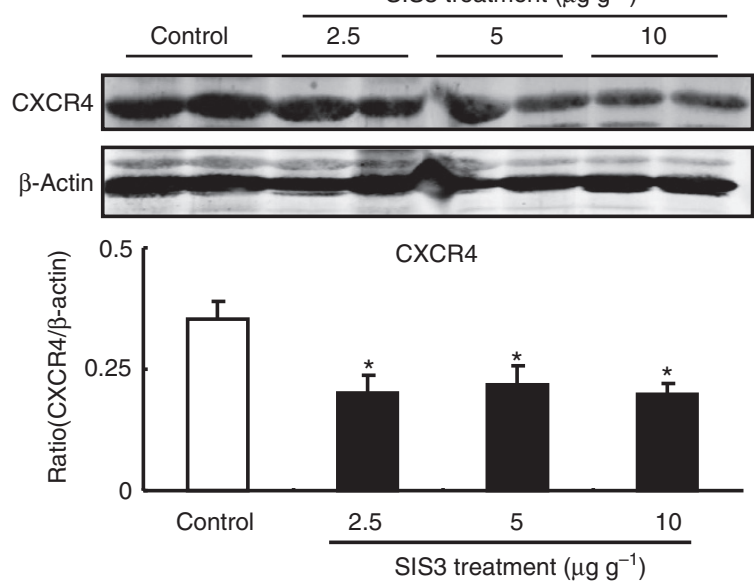

Figure 7 | SIS3 treatment suppresses angiogenesis and the expression of tumour-invasive factors in B16F10 tumour-bearing Smad3 $+/+$ mice. Western blotting analysis shows the intratumoural phosphorylation of Smad3 (a), angiogenesis including the expression of VEGF and CD31 proteins (b), pro- and active MMP-2, MMP-9 and MMP-13 expression (c) and CXCR4 expression (d) of the SIS3-treated mice. Data represent mean \pm s.e.m. for groups of 4-6 mice. ${ }^{\star} P<0.05,{ }^{\star \star} P<0.01,{ }^{\star \star \star} P<0.001$ compared with the control-treated group analysed by analysis of variance.

tumour-suppressive one by targeting the Smad3-dependent tumour microenvironment. We demonstrated that Smad3 is an important checkpoint for TGF- $\beta$-mediated cancer progression in the tumour microenvironment. Therefore, targeting Smad3-dependent tumour microenvironment may represent a novel and effective anticancer therapy.

Several mechanisms may be associated with the promoting effect of Smad3-dependent microenvironment on cancer progression, including angiogenesis, Treg response and tumourinvasive factors, as well as availability of NK cells. We found that genetic deletion or pharmacological inhibition of Smad3 significantly enhanced the production of NK cells $\left(\mathrm{NK} 1.1^{+}\right.$/ $\mathrm{NKp} 46^{+}$cells) locally in the tumour microenvironment and systemically in the peripheral blood and splenic tissue. The effect of $\mathrm{Smad} 3^{-I}$ microenvironment on NK cell production was confirmed on a B16F10-rechallenged model, where increased 
$\mathrm{NK} 1.1^{+} \mathrm{NKp} 46^{+} \mathrm{NK}$ cells were observed in the tumour tissue, blood and spleen of rechallenged Smad3 ${ }^{-1-}$ mice compared with the rechallenged Smad $3^{+9+}$ control. These novel findings suggest that TGF- $\beta$ may act via a Smad3-dependent mechanism to facilitate cancer progression by suppressing NK cell production. This was further confirmed by in vitro finding that deletion of Smad3 also enhanced the production of NKp46 ${ }^{+}$IFN $-\gamma^{+}$NK cells from bone marrow cells. The critical role of NK cell immunity in Smad3-dependent tumour microenvironment was further demonstrated by depleting NK cells from tumour-bearing Smad $3^{-1-}$ mice with a neutralizing anti-NK1.1 antibody to restore the aggressive progression of melanoma. However, the use of anti-NK1.1 antibody may also deplete NKT cells; therefore, the specific role of Smad3 in NK cell production was further examined by adoptive transfer of Smad $3^{-1-}$ or Smad $3^{+1+} \mathrm{NK}$ cells $\left(\mathrm{NK} 1.1^{+}\right)$into the tumour-bearing NK cell deficiency mice (NOD/SCID). We demonstrated an enhanced anticancer effects of Smad $3^{-1-}$ NK cells in cancer progression whereby mice infused with Smad $3^{-1-}$ NK1.1 ${ }^{+}$cells displayed reduced tumour growth compared with Smad3 ${ }^{+} l+$ NK1.1 ${ }^{+}$cell-recipient mice. It has been reported that TGF- $\beta$ can inhibit NK cell-mediated anticancer immunity, including maturation, cytotoxicity, IFN- $\gamma$ production and the expression levels of activating receptors NKG2D and NKp46 (refs 15-17,30-34). TGF- $\beta$ can also suppress CD16-mediated NK cell IFN- $\gamma$ production and antibodydependent cellular cytotoxicity in human NK cells via Smad3 (refs 17). Our findings reveal an unexplored role of Smad3 in the suppression of NK cell production and activities. Results from this study may also provide insights to explain the observation that NK cell development and activities are largely impaired in patients with cancer ${ }^{34-36}$.

Another novel finding from this study was that TGF- $\beta 1$ can directly inhibit the NK cell immunity via a Smad3-E4BP4 axis and facilitate the cancer progression. E4BP4 (NFIL3) is one of the important master transcription factors for NK cell differentiation ${ }^{22-24}$. Here we found that TGF- $\beta 1$ downregulated E4BP4 expression in the bone marrow- and spleen-derived NK cells in a Smad3-dependent manner, whereas genetic deletion or pharmacological inhibition of Smad3 successfully inhibited the TGF- $\beta 1$-mediated E4BP4 suppression in NK cells. More importantly, we identified that E4BP4 is a direct Smad3 target gene. The conserved SBS located in the UTR of E4BP4 gene was validated by ChIP assay. Smad3 could physically bind to the E4BP4 gene and negatively regulate its transcription in response to TGF- $\beta 1$, which was further confirmed by the real-time PCR and luciferase reporter assays. The Smad3-mediated suppression of E4BP4 was functionally important not only in NK cell development but also in the cancer-killing activity. Knockdown of E4BP4 decelerated the differentiation of both CD244 ${ }^{+} \mathrm{NKp} 46^{+}$ (immature $\mathrm{NK}$ cells) and $\mathrm{CD}_{244^{+}}{\mathrm{NKp} 46^{-}}^{-}$(NK cell progenitors) and also reduced the expression of INF- $\gamma$ of NK cells from either Smad3 $3^{-1-}$ or Smad3 $3^{+1+}$ bone marrow cells. Interestingly, comparing to the study with transfer of Smad3 $3^{-1-}$ T-bet knockdown NK cells, adoptive transfer of Smad3 ${ }^{-1-}$ E4BP4 knockdown NK cells significantly promoted tumour growth in NOD/SCID mice with lower levels of IFN- $\gamma$ production. This suggested that E4BP4 may be a more important transcriptional factor than T-bet in NK cell development and functions. The ability of adoptive transfer of E4BP4 knockdown $\mathrm{Smad}^{-1-} \mathrm{NK}$ cells to reverse the suppressive effect of Smad $3^{-1-}$ NK cells on tumour growth in B16F10 tumour-bearing NOD/SCID mice revealed the specific role of E4BP4 in NK cell-mediated antitumour activities. This finding is consistent with a previous study that deletion of T-bet did not alter the IFN- $\gamma$ expression by NK cells in response to parasitic infection ${ }^{37}$. A direct E4BP4-binding site can be predicted on the promoter of IFN- $\gamma$ and the transcription of IFN- $\gamma$ can be reduced in Smad3 $3^{-I-}$ NK cells by E4BP4 knockdown in a T-bet independent manner. Therefore, it is suggested that E4BP4 may be one of the possible candidates of T-bet-independent pathway for Smad3-mediated NK cell immunity. Our study demonstrated that Smad3-mediated inhibition of E4BP4 transcription may be a critical mechanism by which deletion of Smad3 promotes NK cell immunity and suppresses cancer progression. Nevertheless, further investigations should be done to confirm the T-bet independent pathway of TGF- $\beta / S$ mad3-mediated suppression on NK cell immunity.

Interestingly, we found that a majority of NK cells in the Smad3 ${ }^{-l-}$ tumour microenvironment originated from bone marrow, as significant induction of $\mathrm{GFP}^{+}{ }^{\mathrm{NK} 1.1^{+}} \mathrm{NK}$ cells was observed in the tumour tissues of the chimeric $\mathrm{GFP}^{+}$ Smad $3^{-1-}$ mice. Bone marrow is a primary site for NK cell development, and it offers the cellular substrates and soluble factors required for NK cell maturation ${ }^{38}$. NK cells can migrate from the bone marrow to peripheral lymphoid organs, such as the spleen and lymph nodes ${ }^{38}$. NK cells exert promising anticancer activity, which may be circumvent by tumour-friendly microenvironment via TGF- $\beta / S \operatorname{mad} 3$ signalling as defined in the present work. In line with this notion, we found that cancer cells (B16F10 and LLC) proliferated at the same rate in both Smad3 ${ }^{+I+}$ and Smad3 ${ }^{-I-}$ mice during the first week after tumour implantation, but the progression was dramatically inhibited in the Smad $3^{-1-}$ mice from week 2 onwards. This interesting phenomena implies that the host anticancer immunity, presumably NK cells derived from bone marrow, was exclusively suppressed in the $\operatorname{Smad} 3+1+$ mice after the tumour was fully established; but this suppressive effect can be abolished when Smad3 was deleted or inhibited in the tumour microenvironment. This may explain the dramatic increase in $\mathrm{GFP}^{+}$NK1.1 ${ }^{+} \mathrm{NK}$ cells at the tumour stroma of the $\mathrm{GFP}^{+}$Smad3 ${ }^{-1-}$ chimeric mice. These findings also indicate that NK cell development can be strongly suppressed in the bone marrow compartment of $\mathrm{Smad}^{+1+}$ microenvironment during cancer progression, but it can be restored if Smad3 is eliminated. Thus Smad3 may function as a determining factor for $\mathrm{NK}$ cell development in the bone marrow, possibly via the TGF- $\beta 1 / \mathrm{Smad} 3-\mathrm{E} 4 \mathrm{BP} 4$ axis as discovered in this study.

Besides the enhanced NK cell immunity, we also observed that upregulation of angiogenesis markers (for example, VEGF and $\mathrm{CD} 31^{+}$vessels) and cancer invasive and metastatic proteins (for example, MMP-2, MMP-9, MMP-13 and CXCR4) were largely inhibited in the tumour microenvironment of Smad $3^{-1-}$ mice and in Smad $3^{+1+}$ mice treated with SIS3. These findings were consistent with the reported properties of TGF- $\beta / S \operatorname{Smad} 3$ signalling in the induction of VEGF and MMP expression ${ }^{39,40}$. It is well documented that both angiogenesis and cancer invasive and metastatic activities play a critical role in cancer progression ${ }^{41-43}$. Therefore, inhibition of angiogenesis and tumour-invasive activities may account for the additional anticancer mechanism by disrupting $\mathrm{Smad} 3$ in the tumour microenvironments. Furthermore, inhibition of $\mathrm{CD}^{+}{ }^{+}$Foxp $3{ }^{+}$Treg cells could be another mechanism associated with enhanced NK cell immunity in the tumour-bearing Smad $3^{-1-}$ mice. It is well accepted that Smad3 is an important transcriptional factor that binds to Foxp3 and facilitates Treg development ${ }^{12,13}$. It is also reported that Treg cells within the tumour microenvironment can suppress cytotoxicity of NK cells, which is reversed by Treg targeted treatment ${ }^{44,45}$. Thus it is likely that enhancement of NK cellmediated anticancer immunity may be associated with inhibition of Treg cells in the Smad3 $3^{-1-}$ tumour microenvironment. However, the precise mechanism needs to be further investigated. 
Moreover, the present study also demonstrated that targeting Smad3-dependent microenvironment may represent a novel and effective anticancer therapy. By using a SIS3 that specifically inhibits phosphorylation, DNA binding and protein interaction of Smad $3^{29}$, we demonstrated that systemic treatment with SIS3 (2.5, 5, $10 \mu \mathrm{gg}^{-1}$, intraperitoneal (i.p.), daily) successfully blocked the Smad3 activation in the tumour microenvironment and significantly suppressed the tumour growth and invasion and associated with a $100 \%$ overall survival rate in the B16F10 and LLC tumour-bearing Smad3 $3^{+1+}$ mice. In line with the observations in the Smad3 $3^{-1-}$ mice, SIS3 treatment also markedly enhanced the development and anticancer activities of NK cells via a E4BP4-dependent mechanism. This was supported by the finding that SIS3 treatment was capable of increasing E4BP4 expression in Smad3 ${ }^{+1+}$ bone marrow and NK cells and reversed the suppressive effect of Smad3 on NK cell development. In addition, the suppressive effect of SIS3 on cancer progression was also associated with inhibition of angiogenesis (CD31 and VEGF) and tumour-invasive and metastatic activities, including the expression of MMP-2, MMP-9, MMP-13 and CXCR4. It is also possible that direct cytotoxicity of SIS3 on cancer cells in vitro may account for an additional antitumour effect of SIS3 in vivo. The findings suggested SIS3 as a potential checkpoint inhibitor for cancer. Thus systemic Smad3-targeted therapy may represent a novel and effective therapeutic strategy for invasive cancer.

In conclusion, Smad3-dependent tumour microenvironment plays a crucial role in cancer growth, invasion and metastasis. TGF- $\beta$ establishes its tumour-friendly microenvironment by suppressing NK cell production and cancer-killing activity via a Smad3-E4BP4 axis. Our findings suggest that modulating the TGF- $\beta$-dependent tumour microenvironments by targeting Smad3 may represent an effective anticancer therapy.

\section{Methods}

Establishment of LLC-luc and B16F10-luc tumour cell lines. The murine lung adenocarcinoma LLC (CRL-1642, ATCC) and melanoma B16F10 (CRL-6475, ATCC) cells were cultured in DMEM supplemented with $10 \%$ heat-inactivated FCS (GIBCO-BRL), $1 \mu \mathrm{M}$ sodium pyruvate, $2 \mathrm{mM}$ glutamine, $100 \mathrm{U} \mathrm{ml}^{-1}$ penicillin $\mathrm{G}$ and $100 \mu \mathrm{g} \mathrm{ml}^{-1}$ streptomycin. The luciferase overexpression stable cell lines of LLC-luc and B16F10-luc were established by transfection of pRRL-CMV lentiviral-luciferase vector into the cancer cells, followed by clone selection.

Syngeneic mouse tumour models. Littermate $\operatorname{Smad} 3^{-1-}$ (deletion of exon 8 and disruption of exon 7) or Smad3 $+1+$ mice on C57BL/6J $(\mathrm{H}-2 \mathrm{~b})$ background (both sexes, aged 6-8 weeks, 20-25 g) were kindly provided by Dr Chuxia Deng ${ }^{10}$ and were used in this study. Two syngeneic mouse tumour models were induced in Smad $3^{-I-}$ and Smad3 $3^{+I+}$ mice by subcutaneously (s.c.) inoculating $2 \times 10^{6}$ LLC-luc or B16F10-luc cells (both from C57BL/6J mice) into the right flank of the mouse. For tumour rechallenge model, first B16F10 tumour were induced in Smad $3^{-I-}$ and Smad3 ${ }^{+I+}$ mice by s.c. inoculating $1 \times 10^{6}$ B16F10-luc cells into the left flank of the mice, and the established tumours were surgically removed on Day 15. Three weeks after, second B16F10 tumours $\left(1 \times 10^{6}\right.$ cells per mice, s.c.) were induced on the right flank of the same mice, tumour volumes were monitored and samples were collected on Days 15 and 20 for further analysis according to the protocol of Zaharoff et al. ${ }^{46}$. For metastasis study, $3 \times 10^{5}$ of LLC-luc or B16F10-luc cells were i.v. administered into Smad3 $3^{-1-}$ and Smad $3^{+1+}$ mice. The number and volume of surface nodules of B16F10 melanoma (dark black) in the lung of each mouse were counted, while LLC-luc tumours were observed by bioluminescence imaging with IVIS Spectrum system (Caliper, Xenogen). The photon intensity from specific regions was quantified by using the Living Imaging software 4.2 (PerkinElmer). The tumour size was monitored every week with a Vernier caliper and tumour volume was calculated by the formula: $V\left(\right.$ in $\left.\mathrm{mm}^{3}\right)=0.5\left(a b^{2}\right)$, where $a$ is the long diameter and $b$ is the short diameter as previously described ${ }^{47}$. The raw data underlying the tumour volume measurements are shown in Supplementary Data 1. Mice were observed daily for the weight loss, distress, food intake and activities. Mice were killed before the end point if $>20 \%$ weight loss occurred or the tumour reached the maximum permitted size $\left(2,000 \mathrm{~mm}^{3}\right)$, but experiments could be extended under veterinary supervision under special circumstances, for example, if tumours grew faster than expected. The specific experimental approach and the data obtained have been reviewed by the Animal Ethics Experimental Committee at the Chinese University of Hong
Kong and confirmed to be in accordance with local regulations. The survival rate was recorded over the entire experimental period and converted into prolonged survival following the Kaplan-Meier method ${ }^{48}$. All experimental procedures were approved by the Animal Ethics Experimental Committee at the Chinese University of Hong Kong (No. 13/049/GFR).

Tumour model in GFP-expressing bone marrow chimeric mice. To determine the origin and role of Smad3-dependent tumour microenvironments in cancer progression, $\mathrm{GFP}^{+} \mathrm{Smad}^{-1-}$ and $\mathrm{GFP}^{+} \mathrm{Smad} 3^{+1+}$ bone marrow-chimeric mice were generated by cross-breeding Smad3 $+1-$ C57BL/6J mice with GFP transgenic C57BL/6J mice. A lethal irradiation (1500 cGy, Cs-irradiator) were applied on Smad3 $3^{+1+}$ mice for bone marrow depletion and then $5 \times 10^{6}$ of bone marrow cells from the GFP ${ }^{+} \mathrm{Smad}^{-1-}$ or $\mathrm{GFP}^{+} \mathrm{Smad} 3+1+$ mice were i.v. administered into each mice 3 days after irradiation. The establishment of bone marrow chimera was confirmed at week 6 by flow cytometry analysis with $95 \%$ of peripheral blood cells being $\mathrm{GFP}^{+}$cells. We then induced mouse tumour models in $\mathrm{GFP}^{+} \mathrm{Smad}^{-1-}$ and $\mathrm{GFP}^{+} \mathrm{Smad} 3^{+1+}$ mice by s.c. inoculating $2 \times 10^{6}$ LLC-luc or B16F10-luc cells into the right flank of the mouse as described above. The raw data underlying the tumour volume measurements are shown in Supplementary Data 1 . The animals were observed daily and the tumour size and survival rate were measured as described in the section on syngeneic tumour models. The experimental procedures were approved by the Animal Ethics Experimental Committee at the Chinese University of Hong Kong (No. 13/049/GFR).

NK cell depletion and adoptive transfer models. For depletion of NK cells, Smad $3^{+I+}$ and Smad3 ${ }^{-I-}$ mice were pretreated with the anti-NK1.1 (PK136, BioXCell) or immunoglobulin G1 (IgG1) as negative control antibody ( $200 \mu \mathrm{g}$ per mouse, i.p.) at Day -1 . Then B16F10-luc cells $\left(2 \times 10^{6}\right.$ cells $)$ were s.c. inoculated into groups of five mice with or without $\mathrm{NK} 1.1^{+}$cell depletion on Day 0, followed by three additional antibody treatments $(200 \mu \mathrm{g}$ per mouse, i.p., weekly). The efficiency of NK cell depletion was confirmed with the absence of CD49b ${ }^{+}$cells (staining with anti-CD49b antibody, DX5, eBioscience) by flow cytometric analysis of small blood samples collected from the tail vein of antibody-treated mice.

To determine the specific role of Smad3-dependent NK cell antitumour activities, a B16F10 tumour-bearing NOD/SCID mouse model ${ }^{49,50}$ was adoptively transferred with Smad3 ${ }^{+I+}$ or Smad3 ${ }^{-I-}$ NK cells $\left(\mathrm{NK} 1.1^{+}\right)$. Briefly, B16F10 cells $\left(1 \times 10^{6}\right.$ cells) were inoculated s.c. into groups of three NOD/SCID mouse in which both NK and NK T cells are deficient. After tumour establishment on Day 5,

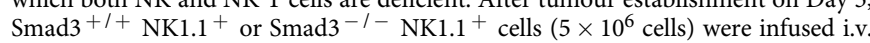
into the B16F10 tumour-bearing NOD/SCID mouse every 5 days with IL-2 supplement (i.p., 1,000 U per mouse) every 2 days for examining tumour growth and NK cell antitumour activities on Day 10.

In addition, we also compared the relative antitumour activities of E4BP4 versus T-bet-dependent NK cells by adoptive transfer of siE4BP4-Smad3 ${ }^{-1-} \mathrm{NK} 1.1^{+}$or siT-bet-Smad $3^{-/-}$NK1.1 ${ }^{+}$NK cells $\left(5 \times 10^{6}\right.$ cells) into B16F10 tumour-bearing NOD/SCID mice as described above for examining tumour growth and NK cell antitumour activities. The raw data underlying the tumour volume measurements are shown in Supplementary Data 1. The animals were observed daily and the tumour size and survival rate were measured as described in the section on syngeneic tumour models. All experimental procedures were approved by Animal Ethics Experimental Committee at the Chinese University of Hong Kong (No. 13/049/GFR and 13/025/MIS).

SIS3 treatment. The tumour-bearing Smad $3+1+$ mice were randomly divided into four groups ( $n=8$ each group) on Day 7 after LLC-luc or B16F10-luc cancer cell (s.c.) inoculation. SIS3 (S0447, Sigma) at different dosages $\left(2.5,5\right.$ or $10 \mu \mathrm{gg}^{-1}$, i.p., daily) were administered into the mice for 2 weeks, whereas control group were received solvent control $(0.05 \%$ dimethyl sulfoxide) instead. Tumour size was monitored with a Vernier caliper every 2 day during the treatment. All mice were subjected to bioluminescence imaging and then killed after the survival rate was recorded on 15 days after SIS3 treatment. To determine the role of NK cells in SIS3-mediated tumour suppression, NK cell depletion was done on SIS3-treated mice and compared the result with SIS3-treated IgG control group on Day 15. The tumours were isolated, weighted and photo-recorded. Tumour tissues were collected for further analysis. The raw data underlying the tumour volume measurements are shown in Supplementary Data 1. The animals were observed daily and the tumour size and survival rate were measured as described in the section on syngeneic tumour models. All experimental procedures were approved by Animal Ethics Experimental Committee at the Chinese University of Hong Kong (No. 13/025/MIS).

NK cell isolation. Splenic and bone marrow NK cells of the tumour-bearing mice were harvested by passing the cells through a $40 \mu \mathrm{m}$ nylon mesh to produce a single-cell suspension. Erythrocyte contamination was eliminated by using RBC lysis buffer (Sigma) and the NK cells were isolated by using the NK Cell Isolation Kit II (Miltenyi Biotec), which provided $90-95 \%$ purity of NK cells as determined by flow cytometry with $\mathrm{CD}^{-}{ }^{-} \mathrm{CD} 49 \mathrm{~b}^{+}$cells. 
Flow cytometric analysis. For quantitative analysis of NK cell populations, tissue samples of the tumour-bearing mice were mechanically dissociated in chilled PBS (supplemented with $3 \% \mathrm{BSA}$ ) and gently mashed through a $40-\mu \mathrm{m}$ nylon mesh to produce a single-cell suspension. NK cell populations were analysed with a flow cytometer by staining with Alexa 488-conjugated anti-mouse CD49b (DX5, eBioscience), PE-conjugated anti-mouse NK1.1 (PK136, eBioscience), Cy3-conjugated anti-mouse NKp46 (bs-2417R-cy3, Bioss) and PE-conjugated anti-mouse CD244 (12-2441-83, eBioscience) antibodies. For IFN- $\gamma$ analysis, NK cells $\left(1 \times 10^{6}\right)$ were cultured in the presence of $2 \mu \mathrm{M}$ monensin (eBioscience) and then fixed and stained intracellular IFN- $\gamma$ (eBioscience) for $5 \mathrm{~h}$. The samples were subjected to acquire on FACSCalibur (Becton Dickinson) and analysed with the WinMDI 2.9 software and Cytobank platform (cytobank.org).

NK cell cytotoxicity assay. The anticancer activity of NK cells was evaluated by using a Cella-Tox Bioluminescence Cytotoxicity Assay Kit (Cell Technology Inc.). In brief, isolated splenic NK cells from the tumour-bearing Smad3 $3^{-1-}$ or Smad $3^{+1+}$ mice were incubated with the NK-sensitive target cells (YAC-1), syngeneic LLC or B16F10 cells at ratios of 1.25:1, 2.5:1, 5:1, 10:1 and 20:1, respectively. In addition, splenic NK cells isolated from normal Smad $3{ }^{+1+}$ mice activated by $500 \mathrm{U} \mathrm{ml}^{-1} \mathrm{IL}-2$ for 3 days with or without SIS3 treatment or Day -8 Smad $3^{-I}$ or Smad $3{ }^{+I+}$ (with or without SIS3 treatment) bone marrow-derived NK cells were incubated with B16F10 cells. After 4 -h incubation at $37^{\circ} \mathrm{C}$, cytotoxicity signals were detected by a luciferase bioluminescence method, and results were calculated according to the manufacturer's instructions.

Western blotting analysis. Total proteins of tumour or normal (the skin of the same mouse) tissues were extracted by chilled RIPA lysis buffer (Pierce) and then subjected to the western blotting analysis with primary antibodies against CD31, VEGF, MMP-2, MMP-9, MMP-13, CXCR4, NKp46, p-Smad3, Smad3 (all from Santa Cruz Biotechnology) and E4BP4 (Cell Signaling) in 1:1,000, followed by incubation with the corresponding IRDyeTM800-conjugated secondary antibodies $(1: 10,000$, Rockland Immunochemicals). $\beta$-Actin was used as an internal control. Expression levels of the proteins were detected by using LiCor/Odyssey infrared image system (LI-COR; Biosciences), and the band intensities were quantified with the Image J software (version 1.48, NIH, Bethesda). Images have been cropped for presentation; their full size images are presented in Supplementary Figs 15-20.

Histology and immunohistochemistry. For immunohistochemistry, tumour tissues embedded in Optimal Cutting Temperature were cut into $6 \mu \mathrm{M}$ sections and stained with the specific antibodies. The microvessels of tumour tissues were detected by anti-mouse CD31 antibody (BD Pharmingen) with Alexa Fluor 488-conjugated secondary antibody (Invitrogen), and the results were quantified as microvessel densities according to the method of Weidner et al. ${ }^{51}$. Treg cells in the tumour tissues were detected by immunofluorescence with fluorescein isothiocyanate (FITC)-conjugated anti-CD4 and Cy3-conjugated anti-Foxp3 antibodies (eBioscience). NK cells were identified by anti-mouse NK1.1 (PK136; $\mathrm{R} \& \mathrm{D})$ and NKp46 (R\&D) antibodies, while IFN- $\gamma$ expressing NK cells were determined with Cy3- or APC-conjugated anti-NK1.1, FITC-conjugated anti-NKp46 and FITC- or APC-conjugated anti-IFN- $\gamma$ antibodies (eBioscience). All antibodies were 1:100 in dilution. Cell nuclei were counterstained with DAPI (4,6-diamidino-2-phenylindole; Sigma). Positive cells and total cell counts from 10 high-power-field $(\times 40)$ tumour tissues were scored and expressed as a percentage of positive cells for each animal.

Enzyme-linked immunosorbent assay. Levels of plasma and intratumoural granzyme B, IL-2 and IFN- $\gamma$ were determined by using the commercial enzymelinked immunosorbent assay kits (eBioscience). To collect the intratumoural tissue fluids, the chilled PBS was added into each tumour tissue samples in a ratio of $100 \mathrm{mg}$ tissue per $\mathrm{ml}$. The tissues were then homogenized and centrifuged at 14,000 r.p.m. at $4{ }^{\circ} \mathrm{C}$ for $10 \mathrm{~min}$. The collected tumour tissue solutions and blood plasma were subjected for determination of NK cell activities, including granzyme $\mathrm{B}, \mathrm{IL}-2$ and IFN- $\gamma$, following the manufacturer's instruction.

RNA extraction and real-time PCR. Total RNA was isolated from the cells using the RNeasy Isolation Kit (Qiagen, Valencia, CA) according to the manufacturer's instructions. Real-time PCR was performed using Bio-Rad iQ SYBR Green supermix with Opticon2 (Bio-Rad, Hercules, CA) as described previously ${ }^{52}$. The primers used in this study included: E4BP4 forward 5'-CAGTGCAGGTGACGA ACATT $-3^{\prime}$ and reverse $5^{\prime}$-TGTTCCACCACACCTGTTTTGA- $3^{\prime}$; and GAPDH (glyceraldehyde 3-phosphate dehydrogenase) forward 5'-GCATGGCCTTCCGTG TTC- $3^{\prime}$ and reverse $5^{\prime}$-GATGTCATCATACTTGGCAGGTTT- $3^{\prime}$. The ratio for E4BP4 expression was normalized with GAPDH and expressed as the mean \pm s.e.m.

siRNA knockdown. Smad $3^{-1-}$ and $\operatorname{Smad} 3^{+/+}$bone marrow cells were harvested and transfected with $(100 \mathrm{nM})$ nonsense control (siN05815122147, RIBOBIO) and siRNA against E4BP4 (5'-GAUGAGGGUGUAGUGGGCAAGU
CUU- $3^{\prime}$ ) on Days 0 and 4 as described previously ${ }^{53}$. The bone marrow cells were ex vivo differentiated into NK cells according to the protocol of Fathman et al. ${ }^{54}$ For adoptive transfer studies in NOD/SCID mice model, the ex vivo differentiated NK cells were transfected with nonsense control, siRNA against E4BP4 or T-bet ( $5^{\prime}$-GGGAGAACUUUGAGUCCAU-3') on Day 5 . The cells were collected on Day 6 (for Fig. 7) or Day 7 (for NK cell infusion, Supplementary Fig. 5). The maturation and cytotoxicity of NK cells were quantified by flow cytometry according to the CD244, NK1.1, NKp46 or IFN- $\gamma$ expression profiles with directed dye-conjugated anti-mouse antibodies (eBioscience).

MTT (methyl-thiazoldiphenyl tetrazolium) assay. The MTT assay was used to determine the antiproliferative activity of SIS3 on B16F10 cells in vitro. In brief, cancer cells $\left(1 \times 10^{4}\right.$ per well) were seeded on a 96-well plate and serial concentrations of SIS3 $(0,2.5,5,10,15,20 \mu \mathrm{M})$ were added on the next day. After 24-h of SIS3 treatment, $30 \mu \mathrm{l}$ of MTT ( $5 \mathrm{mg} \mathrm{ml}^{-1}$ ) was added to each well and incubated for $2 \mathrm{~h}$ at $37^{\circ} \mathrm{C}$. The MTT solution was then replaced by $100 \mu \mathrm{l}$ of dimethyl sulfoxide in each well and measured with a microtitre-plate reader (Bio-Rad) at $540 \mathrm{~nm}$, and all data were calculated as a percentage against the control.

ChIP assay. ChIP was performed by using the SimpleChIP Enzymatic Chromatin IP Kit (magnetic beads; Cell Signaling) according to the manufacturer's instructions. In brief, bone marrow cells were isolated from Smad $3+1+$ mice pretreated with $1 \mathrm{ng} \mathrm{ml}{ }^{-1}$ TGF- $\beta 1$ for $2 \mathrm{~h}$ and then isolated by the Kit (Cell Signaling). Immunoprecipitation was performed with the antibody against Smad3 $(1: 100)$ and IgG was used as a control (Cell Signaling). Precipitated DNAs were identified by PCR using specific primers that detect the binding of Smad3 to $3^{\prime}$ UTR of mouse E4BP4 gene: forward $5^{\prime}$-CCTCTGACACATCGGAGAGC- $3^{\prime}$ and reverse $5^{\prime}$-CGG AGAACAAGCTGATCGCCCT- ${ }^{\prime}$.

Luciferase reporter assay of E4BP4 $\mathbf{3}^{\prime}$ UTR. Construction of reporter and mutant plasmids and luciferase assay were performed by Landbiology (Guangzhou, China). For E4BP4 $3^{\prime}$ UTR reporter, a pair of reporter plasmids was constructed with the SBS in E4BP4 $3^{\prime}$ UTR or its mutant by inserting the following oligos (E4BP4XhoIF: $5^{\prime}$-ccgctcgagATGGCTGCTGACCGAGCTATGC-3' and E4BP4NotIR:5' -ataagaatgcggccgcTGTGCTTGCCACCAATCCTTT-3') or (mut-E4BP4_F:5'-GATGCCCTCACTCTGCCTGGCGTGTAAATTTGGGGCCC CCCACAGAGGCTGTACATACT-3' ${ }^{\prime}$ and mut-E4BP4_R:5' -AGTATGTACAGCC TCTGTGGGGGGCCCCAAATTTACACGCCAGGCAGAGTGAGGGCATC-3') into the psiCHECK-2 vector. In addition, a pair of overexpression plasmids was constructed with the complete cds of Smad3 (GI:2564492) or its mutant with the deletion of exon 8 by amplifying our Smad3 full-length pcDNA3.0 plasmid with the following primers: (mut-Smad3_F:5'-TGGGTTCCCCCAGCATCCGCTGTTC CGTGTAGAGACACTGGGAGTAAAGGGATCGGGT- ${ }^{\prime}$ and mut-Smad3_R: 5'-ACCCGATCCCTTTACTCCCAGTGTCTCTACACGGAACAGCGGATGCT GGGGGAACCCA-3').

For dual luciferase reporter assay (Promega), the E4BP4 luciferase reporter plasmids and Smad3 plasmids were co-transfected into 293T cells. The luminescence in lysates of harvested cells was measured at $48 \mathrm{~h}$ according to the manufacturer's instructions. The luciferase activity (M1) and Renilla luciferase activity (M2) were measured by GloMax-Multi Detection System (Promega). The reporter activity was represented by the ratio of M1/M2, which was the normalized luciferase activity of the experimental plasmid (pGL4-basic; Promega). Results are shown as mean \pm s.d. fold induction of luciferase in at least three independent experiments.

Statistical analysis. Statistical analysis of the differences in MTT assay, tumour volume, tumour weight, photon intensity, ratios of tumour nodules and positive cells, protein and mRNA expression levels, dual luciferase reporter assay and NK cell cytotoxicity were performed using analysis of variance, followed by Newman-Keuls Post Test from the Prism Program (Prism 5.0 GraphPad Software, San Diego, CA). Survival rates were generated based on the Kaplan-Meier method and statistical significance was determined by the log-rank test, $P$ value $<0.05$ was considered statistically significant.

Data availability. All data are available within the article, as figure source data or Supplementary Information Files, or from the authors upon reasonable request.

\section{References}

1. Schreiber, R. D., Old, L. J. \& Smyth, M. J. Cancer immunoediting: integrating immunity's roles in cancer suppression and promotion. Science 331, 1565-1570 (2011).

2. Junttila, M. R. \& de Sauvage, F. J. Influence of tumour micro-environment heterogeneity on therapeutic response. Nature 501, 346-354 (2013).

3. Derynck, R., Akhurst, R. J. \& Balmain, A. TGF- $\beta$ signaling in tumor suppression and cancer progression. Nat. Genet. 29, 117-129 (2001).

4. Massagué, J. TGFbeta in cancer. Cell 134, 215-230 (2008). 
5. Ikushima, H. \& Miyazono, K. TGFbeta signalling: a complex web in cancer progression. Nat. Rev. Cancer 10, 415-424 (2010).

6. Pickup, M., Novitskiy, S. \& Moses, H. L. The roles of TGF $\beta$ in the tumour microenvironment. Nat. Rev. Cancer 13, 788-799 (2013).

7. Yang, L., Pang, Y. \& Moses, H. L. TGF-beta and immune cells: an important regulatory axis in the tumor microenvironment and progression. Trends Immunol. 31, 220-227 (2010).

8. Flavell, R. A., Sanjabi, S., Wrzesinski, S. H. \& Licona-Limón, P. The polarization of immune cells in the tumour environment by TGFbeta. Nat. Rev. Immunol. 10, 554-567 (2010).

9. Datto, M. B. et al. Targeted disruption of Smad3 reveals an essential role in transforming growth factor beta-mediated signal transduction. Mol. Cell Biol. 19, 2495-2504 (1999).

10. Yang, X. et al. Targeted disruption of SMAD3 results in impaired mucosal immunity and diminished $\mathrm{T}$ cell responsiveness to TGF-beta. EMBO J. 18, 1280-1291 (1999).

11. Giroux, M. et al. SMAD3 prevents graft-versus-host disease by restraining Th1 differentiation and granulocyte-mediated tissue damage. Blood 117, 1734-1744 (2011).

12. Schlenner, S. M., Weigmann, B., Ruan, Q., Chen, Y. \& von Boehmer, H. Smad3 binding to the foxp3 enhancer is dispensable for the development of regulatory $\mathrm{T}$ cells with the exception of the gut. J. Exp. Med. 209, 1529-1535 (2012).

13. Martinez, G. J. et al. Smad3 differentially regulates the induction of regulatory and inflammatory T cell differentiation. J. Biol. Chem. 284, 35283-35286 (2009).

14. Gu, A. D., Wang, Y., Lin, L., Zhang, S. S. \& Wan, Y. Y. Requirements of transcription factor Smad-dependent and -independent TGF- $\beta$ signaling to control discrete T-cell functions. Proc. Natl Acad. Sci. USA 109, 905-910 (2012).

15. Rook, A. H. et al. Effects of transforming growth factor beta on the functions of natural killer cells: depressed cytolytic activity and blunting of interferon responsiveness. J. Immunol. 136, 3916-3920 (1986).

16. Bellone, G., Aste-Amezaga, M., Trinchieri, G. \& Rodeck, U. Regulation of NK cell functions by TGF-beta 1. J. Immunol. 155, 1066-1073 (1995).

17. Trotta, R. et al. TGF-beta utilizes SMAD3 to inhibit CD16-mediated IFNgamma production and antibody-dependent cellular cytotoxicity in human NK cells. J. Immunol. 181, 3784-3792 (2008).

18. Zhu, Y., Richardson, J. A., Parada, L. F. \& Graff, J. M. Smad3 mutant mice develop metastatic colorectal cancer. Cell 94, 703-714 (1998).

19. Maggio-Price, L. et al. Helicobacter infection is required for inflammation and colon cancer in SMAD3-deficient mice. Cancer Res. 66, 828-838 (2006).

20. Maggio-Price, L. et al. Bacterial infection of Smad3/Rag2 double-null mice with transforming growth factor-beta dysregulation as a model for studying inflammation-associated colon cancer. Am. J. Pathol. 174, 317-329 (2009).

21. Li, A. G., Lu, S. L., Zhang, M. X., Deng, C. \& Wang, X. J. Smad3 knockout mice exhibit a resistance to skin chemical carcinogenesis. Cancer Res. 64, 7836-7845 (2004).

22. Male, V. et al. The transcription factor E4bp4/Nfil3 controls commitment to the NK lineage and directly regulates Eomes and Id2 expression. J. Exp. Med. 211, 635-642 (2014).

23. Kamizono, S. et al. Nfil3/E4bp4 is required for the development and maturation of NK cells in vivo. J. Exp. Med. 206, 2977-2986 (2009).

24. Gascoyne, D. M. et al. The basic leucine zipper transcription factor E4BP4 is essential for natural killer cell development. Nat. Immunol. 10, 1118-1124 (2009).

25. Lo, R. S. \& Witte, O. N. Transforming growth factor-beta activation promotes genetic context-dependent invasion of immortalized melanocytes. Cancer Res. 68, 4248-4257 (2008).

26. Du, G. et al. Naringenin: a potential immunomodulator for inhibiting lung fibrosis and metastasis. Cancer Res. 69, 3205-3212 (2009).

27. Ovcharenko, I., Nobrega, M. A., Loots, G. G. \& Stubbs, L. ECR Browser: a tool for visualizing and accessing data from comparisons of multiple vertebrate genomes. Nucleic Acids Res. 32, W280-W286 (2004).

28. Shultz, L. D. et al. Multiple defects in innate and adaptive immunologic function in NOD/LtSz-scid mice. J. Immunol. 154, 180-191 (1995).

29. Jinnin, M., Ihn, H. \& Tamaki, K. Characterization of SIS3, a novel specific inhibitor of Smad3, and its effect on transforming growth factorbetal-induced extracellular matrix expression. Mol. Pharmacol. 69, 597-607 (2006).

30. Laouar, Y., Sutterwala, F. S., Gorelik, L. \& Flavell, R. A. Transforming growth factor- $\beta$ controls $\mathrm{T}$ helper type 1 cell development through regulation of natural killer cell interferon- $\gamma$. Nat. Immunol. 6, 600-607 (2005).

31. Lee, J. C., Lee, K. M., Kim, D. W. \& Heo, D. S. Elevated TGF-betal secretion and down-modulation of NKG2D underlies impaired NK cytotoxicity in cancer patients. J. Immunol. 172, 7335-7340 (2004)
32. Mamessier, E. et al. Human breast cancer cells enhance self tolerance by promoting evasion from NK cell antitumor immunity. J. Clin. Invest. 121, 3609-3622 (2011).

33. Wu, Y. et al. Monocyte/macrophage-elicited natural killer cell dysfunction in hepatocellular carcinoma is mediated by CD48/2B4 interactions. Hepatology 57, 1107-1116 (2013).

34. Wilson, E. B. et al. Human tumour immune evasion via TGF- $\beta$ blocks NK cell activation but not survival allowing therapeutic restoration of anti-tumour activity. PLoS ONE 6, e22842 (2011).

35. Imai, K. et al. Natural cytotoxic activity of peripheral-blood lymphocytes and cancer incidence: an 11-year follow-up study of a general population. Lancet 356, 1795-1799 (2000).

36. Pasero, C. et al. Highly effective NK cells are associated with good prognosis in patients with metastatic prostate cancer. Oncotarget 6, 14360-14373 (2015).

37. Harms Pritchard, G. et al. Diverse roles for T-bet in the effector responses required for resistance to infection. J. Immunol. 194, 1131-1140 (2015).

38. Yu, J., Freud, A. G. \& Caligiuri, M. A. Location and cellular stages of natural killer cell development. Trends Immunol. 34, 573-582 (2013).

39. Nakagawa, T. et al. TGF-beta induces proangiogenic and antiangiogenic factors via parallel but distinct Smad pathways. Kidney Int. 66, 605-613 (2004).

40. Leivonen, S. K. et al. Activation of Smad signaling enhances collagenase-3 (MMP-13) expression and invasion of head and neck squamous carcinoma cells. Oncogene 25, 2588-2600 (2006).

41. Weis, S. M. \& Cheresh, D. A. Tumor angiogenesis: molecular pathways and therapeutic targets. Nat. Med. 17, 1359-1370 (2011).

42. Kessenbrock, K., Plaks, V. \& Werb, Z. Matrix metalloproteinases: regulators of the tumor microenvironment. Cell 141, 52-67 (2010).

43. Bertran, E. et al. Overactivation of the TGF- $\beta$ pathway confers a mesenchymallike phenotype and CXCR4-dependent migratory properties to liver tumor cells. Hepatology 58, 2032-2044 (2013).

44. Ghiringhelli, F. et al. $\mathrm{CD} 4+\mathrm{CD} 25+$ regulatory T cells inhibit natural killer cell functions in a transforming growth factor-beta-dependent manner. J. Exp. Med. 202, 1075-1085 (2005).

45. Jie, H. B. et al. CTLA-4 + Regulatory T cells increased in cetuximab-treated head and neck cancer patients suppress NK cell cytotoxicity and correlate with poor prognosis. Cancer Res. 75, 2200-2210 (2015).

46. Zaharoff, D. A., Hance, K. W., Rogers, C. J., Schlom, J. \& Greiner, J. W. Intratumoral immunotherapy of established solid tumors with chitosan/IL-12 J. Immunother. 33, 697-7052010 (2010).

47. Zhou, S. et al. Combination therapy of VEGF-trap and gemcitabine results in improved anti-tumor efficacy in a mouse lung cancer model. PLOS ONE 8, e68589 (2013).

48. Zhou, S. et al. CCR7 expression and intratumoral FOXP3 + regulatory T cells are correlated with overall survival and lymph node metastasis in gastric cancer. PLoS ONE 8, e74430 (2013).

49. Kataoka, S. et al. Immunologic aspects of the nonobese diabetic (NOD) mouse. Abnormalities of cellular immunity. Diabetes 32, 247-253 (1983).

50. Imamura, M. et al. Autonomous growth and increased cytotoxicity of natural killer cells expressing membrane-bound interleukin-15. Blood 124, 1081-1088 (2014).

51. Weidner, N., Semple, J. P., Welch, W. R. \& Folkman, J. Tumor angiogenesis and metastasis: correlation in invasive breast carcinoma. N. Engl. J. Med. 324, $1-8$ (1991)

52. Meng, X. M. et al. Smad2 protects against TGF-beta/Smad3-mediated renal fibrosis. J. Am. Soc. Nephrol. 21, 1477-1487 (2010).

53. Zhou, Q., Huang, X. R., Yu, J., Yu, X. \& Lan, H. Y. Long noncoding RNA Arid2-IR is a novel therapeutic target for renal inflammation. Mol. Ther. 23, 1034-1043 (2015).

54. Fathman, J. W. et al. Identification of the earliest natural killer cell-committed progenitor in murine bone marrow. Blood 118, 5439-5447 (2011).

\section{Acknowledgements}

We thank Dr Chuxia Deng for Smad3 ${ }^{-1-}$ mice. This study was supported by the Research Grants Council of Hong Kong (GRF 469110, 468711, CUHK3/CRF/12R), Innovation and Technology Fund of Hong Kong (ITS/227/15), the Focused Investment Scheme A from Chinese University of Hong Kong and Hong Kong Scholar Program.

\section{Author contributions}

P.M.-K.T., S.Z. and X.-M.M. have contributed equally to this work. H.-Y.L. designed and supervised all experiments and contributed to manuscript preparation. P.M.-K.T, S.Z. and X.-M.M. performed experiments, analysed data and contributed to manuscript preparation. Q.-M.W., G.-Y.L. and C.-J.L. collected animal samples and participated in animal experiments. X.-R.H., Y.-J.T., X.-Y.G., B.P.Y. and K.-F.T. contributed to data analysis and manuscript preparation. 


\section{Additional information}

Supplementary Information accompanies this paper at http://www.nature.com/ naturecommunications

Competing financial interests: The authors declare no competing financial interests.

Reprints and permission information is available online at http://npg.nature.com/ reprintsandpermissions/

How to cite this article: Tang, P. M.-K. et al. Smad3 promotes cancer progression by inhibiting E4BP4-mediated NK cell development. Nat. Commun. 8, 14677

doi: 10.1038/ncomms14677 (2017).
Publisher's note: Springer Nature remains neutral with regard to jurisdictional claims in published maps and institutional affiliations.

cc (i) This work is licensed under a Creative Commons Attribution 4.0 International License. The images or other third party material in this article are included in the article's Creative Commons license, unless indicated otherwise in the credit line; if the material is not included under the Creative Commons license, users will need to obtain permission from the license holder to reproduce the material. To view a copy of this license, visit http://creativecommons.org/licenses/by/4.0/

(C) The Author(s) 2017 